\title{
Seeds of Life in Space (SOLIS). III. Zooming Into the Methanol Peak of the Prestellar Core L1544*
}

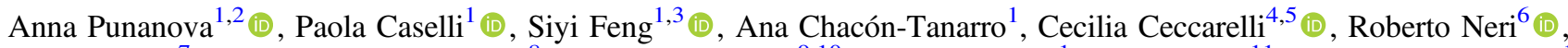

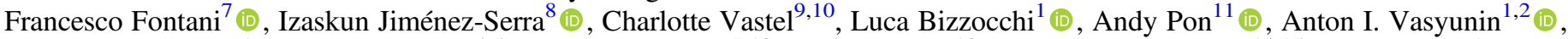

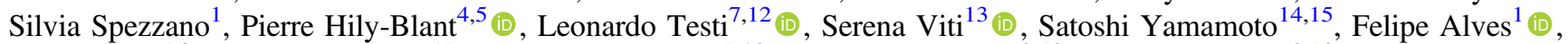
Rafael Bachiller ${ }^{16}$ (D), Nadia Balucani ${ }^{17}$ (10), Eleonora Bianchi ${ }^{7,18}$, Sandrine Bottinelli ${ }^{9,10}$, Emmanuel Caux ${ }^{9,10}$, Rumpa Choudhury ${ }^{1}$, Claudio Codella ${ }^{7}$ (1) , François Dulieu ${ }^{19}$, Cécile Favre ${ }^{7}$ (1) Jonathan Holdship ${ }^{13}$ (10), Ali Jaber Al-Edhari ${ }^{4,5,20}$, Claudine Kahane ${ }^{4,5}$ (1),

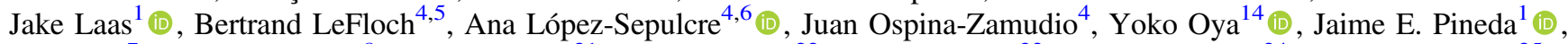
Linda Podio $^{7}$, Davide Quenard ${ }^{8}$, Albert Rimola ${ }^{21}$ (D), Nami Sakai ${ }^{22}$ (1) Ian R. Sims ${ }^{23}$, Vianney Taquet ${ }^{24}$ (i), Patrice Theulé ${ }^{25}$, and

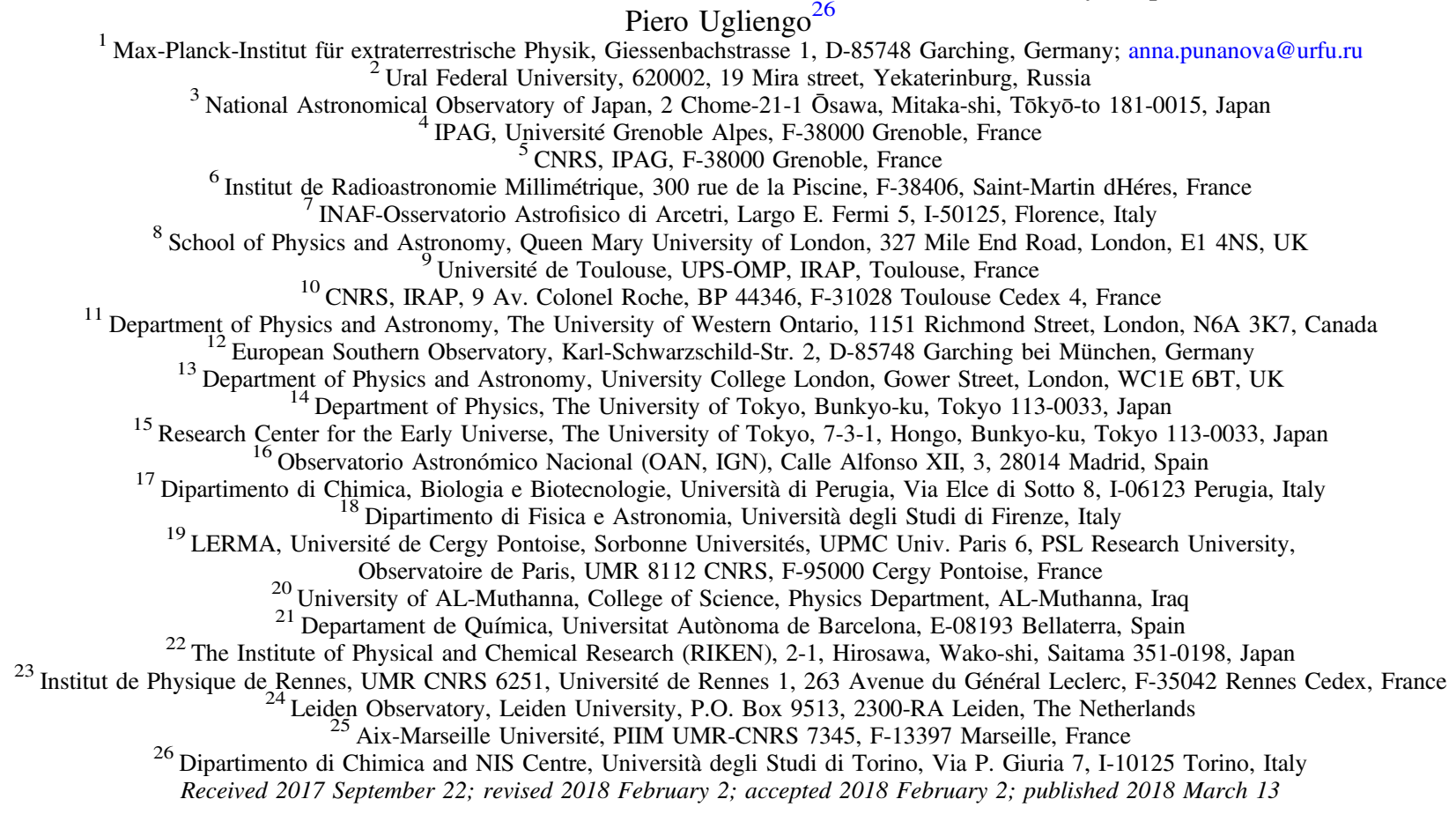
Piero Ugliengo ${ }^{26}$

\begin{abstract}
Toward the prestellar core $\mathrm{L} 1544$, the methanol $\left(\mathrm{CH}_{3} \mathrm{OH}\right)$ emission forms an asymmetric ring around the core center, where $\mathrm{CH}_{3} \mathrm{OH}$ is mostly in solid form, with a clear peak at 4000 au to the northeast of the dust continuum peak. As part of the NOEMA Large Project SOLIS (Seeds of Life in Space), the $\mathrm{CH}_{3} \mathrm{OH}$ peak has been spatially resolved to study its kinematics and physical structure and to investigate the cause behind the local enhancement. We find that methanol emission is distributed in a ridge parallel to the main axis of the dense core. The centroid velocity increases by about $0.2 \mathrm{~km} \mathrm{~s}^{-1}$ and the velocity dispersion increases from subsonic to transonic toward the central zone of the core, where the velocity field also shows complex structure. This could be an indication of gentle accretion of material onto the core or the interaction of two filaments, producing a slow shock. We measure the rotational temperature and show that methanol is in local thermodynamic equilibrium (LTE) only close to the dust peak, where it is significantly depleted. The $\mathrm{CH}_{3} \mathrm{OH}$ column density, $N_{\text {tot }}\left(\mathrm{CH}_{3} \mathrm{OH}\right)$, profile has been derived with non-LTE radiative transfer modeling and compared with chemical models of a static core. The measured $N_{\text {tot }}\left(\mathrm{CH}_{3} \mathrm{OH}\right)$ profile is consistent with model predictions, but the total column densities are one order of magnitude lower than those predicted by models, suggesting that the efficiency of reactive desorption or atomic hydrogen tunneling adopted in the model may be overestimated; or that an evolutionary model is needed to better reproduce methanol abundance.
\end{abstract}

Key words: ISM: clouds - ISM: individual objects (L1544) - ISM: kinematics and dynamics - ISM: molecules radio lines: ISM - stars: formation

\footnotetext{
* This work is based on observations carried out under project number L15AA with the IRAM NOEMA Interferometer and on observations carried out with the IRAM $30 \mathrm{~m}$ telescope. IRAM is supported by INSU/CNRS (France), MPG (Germany), and IGN (Spain).
}

\section{Introduction}

Methanol $\left(\mathrm{CH}_{3} \mathrm{OH}\right)$ is a crucial molecule for the growth of molecular complexity in the interstellar medium, as it is a key precursor for many organic and prebiotic molecules found in 
regions of star and planet formation (e.g., Herbst \& van Dishoeck 2009). Methanol is widespread in our Galaxy and it is present in different environments, such as the molecular envelopes surrounding low-mass and high-mass protostars (the so-called hot corinos and hot cores), and cold dense clouds in low- and high-mass star-forming regions (e.g., Blake et al. 1987; Gibb et al. 2000; Schöier et al. 2002; Parise et al. 2004; Maret et al. 2005; Tafalla et al. 2006; Bizzocchi et al. 2014; Vastel et al. 2014). According to present models and experiments, methanol is formed on dust grains via the hydrogenation of CO (e.g., Tielens \& Hagen 1982; Watanabe \& Kouchi 2002; Rimola et al. 2014) and released to the gas phase via thermal and/or nonthermal processes (Garrod \& Herbst 2006; Vasyunin \& Herbst 2013). In the cold $(\simeq 10 \mathrm{~K})$ dense $\left(10^{4}-10^{7} \mathrm{~cm}^{-3}\right)$ gas of prestellar cores, thermal desorption is not effective and reactive desorption is thought to be responsible for the release of methanol into the gas phase upon formation on icy mantles (e.g., Garrod et al. 2007; Vasyunin \& Herbst 2013), in particular, on CO-rich surfaces (Minissale et al. 2016b; Vasyunin et al. 2017). The photo desorption of methanol is not effective as it breaks the molecule into fragments, as has been shown experimentally (Bertin et al. 2016; Cruz-Diaz et al. 2016).

In dense cold cores, gaseous methanol should preferentially be found in a shell around the dense central regions, where visual extinctions are large enough to screen interstellar UV photons ( $\geqslant 10 \mathrm{mag}$ ) and volume densities are around a few $\times 10^{4} \mathrm{~cm}^{-3}$ (Vasyunin et al. 2017). In these conditions, carbon atoms are mainly locked in $\mathrm{CO}$ molecules and $\mathrm{CO}$ freeze-out becomes significant (Caselli et al. 1999; Tafalla et al. 2002). Methanol is then produced via surface hydrogenation of the frozen $\mathrm{CO}$ molecules and it is partially returned to the gas phase upon formation on CO-rich ices (Vasyunin et al. 2017). At higher densities, i.e., toward the core center, the freeze-out rate of methanol overcomes its production rate, with a consequent drop in its gas-phase abundance (Vasyunin et al. 2017). Thus, gas-phase methanol is expected to be abundant at the edge of the CO-depleted zone. In fact, observations of methanol toward dense cores (L1498, L1517B; Tafalla et al. 2006) reveal ring-like structures.

In this work, we focus on the methanol emission toward L1544. This is a prototypical prestellar core, being centrally concentrated (Ward-Thompson et al. 1999), with a central density of $2 \times 10^{6} \mathrm{~cm}^{-3}$, low central temperatures ranging from 5 to $11 \mathrm{~K}$ in the inner 10,000 au (Crapsi et al. 2007) and undergoing a slow quasi-static contraction (Tafalla et al. 1998; Keto \& Caselli 2010; Keto et al. 2015). It presents the chemical features of $\mathrm{CO}$ freeze-out and enhanced deuteration toward the center (Caselli et al. 1999, 2002b; Vastel et al. 2006). L1544 also shows signs of chemical differentiation, with methanol residing away from the sharp $\mathrm{H}_{2}$ column density drop toward the southeast of the core, rich in carbon chain molecules (Spezzano et al. 2016, 2017). $\mathrm{CH}_{3} \mathrm{OH}$ toward $\mathrm{L} 1544$ has also been found to have an asymmetric ring-like distribution, with the peak located toward the northeast of the dust peak (see Figure 1), away from the low extinction regions (Bizzocchi et al. 2014; Spezzano et al. 2016). Several complex organic molecules (e.g., acetaldehyde, formic acid, dimethyl ether, methyl formate) have been detected toward L1544 (Vastel et al. 2014; Jiménez-Serra et al. 2016). At the location of the methanol peak of L1544, Jiménez-Serra et al. (2016) found enhanced abundances of O-bearing complex organic molecules

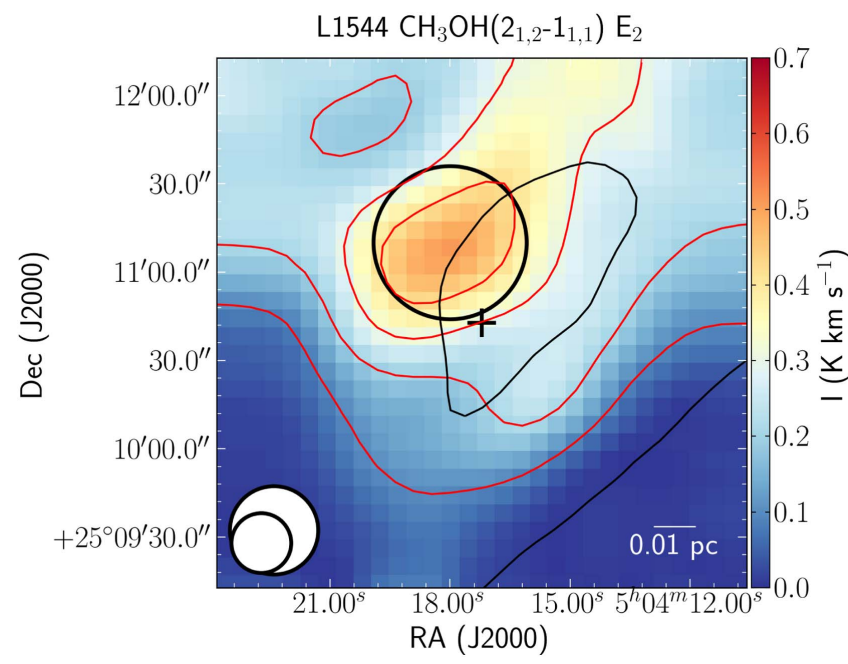

Figure 1. Methanol emission toward L1544 mapped with the IRAM $30 \mathrm{~m}$ antenna (colorscale, Bizzocchi et al. 2014) and the $250 \mu \mathrm{m}$ dust continuum emission mapped with Herschel/SPIRE (black contours, André et al. 2010). The red contours of methanol start at $10 \sigma_{I}\left(0.1 \mathrm{~K} \mathrm{~km} \mathrm{~s}^{-1}\right)$ with a step of $10 \sigma_{I}$. The thin black contours show $50 \%$ and $90 \%$ of the peak dust emission $\left(232.81 \mathrm{MJy} \mathrm{sr}^{-1}\right)$. The thick black circle shows the NOEMA primary beam. The Herschel and the $30 \mathrm{~m}$ beams are shown in the bottom left (the larger beam is of Herschel, the smaller beam is of the $30 \mathrm{~m}$ ). The $1.3 \mathrm{~mm}$ dust continuum emission peak (Ward-Thompson et al. 1999), considered as the core center, is shown with the cross.

(in particular $\mathrm{CH}_{3} \mathrm{CHO}, \mathrm{HCOOCH}_{3}$, and $\mathrm{CH}_{3} \mathrm{OCH}_{3}$ ), likely related to methanol (also $\mathrm{HCO}$, Spezzano et al. 2017), as well as $\mathrm{CH}_{3} \mathrm{O}$, a possible product of methanol photodissociation (Bertin et al. 2016; Cruz-Diaz et al. 2016) or, alternatively, a product of rapid gas-phase reactions between methanol and hydroxyl radical (OH; Shannon et al. 2014).

We present interferometric observations of the methanol peak of L1544, with the aim of investigating its origins. This work is part of the NOEMA (Northern Extended Millimetre Array) large program SOLIS (Seeds of Life in Space), aimed at studying the formation of complex organic molecules across all stages of star formation (Ceccarelli et al. 2017). In Section 2, the details of the observations, the data reduction procedure and Gaussian fitting of the spectra are presented. Section 3 presents the results of the Gaussian fitting, velocity gradients, rotational temperatures, and column density calculations. In Section 4, we discuss the results and possible origins of the methanol-rich zone. The summary of the paper is given in Section 5.

\section{Observations, Data Reduction, and Line Fitting}

\subsection{Observations}

Observations of the $\left(2_{1,2}-1_{1,1}\right)-E_{2}, \quad\left(2_{0,2}-1_{0,1}\right)-A^{+}$, and $\left(2_{0,2}-1_{0,1}\right)-E_{1}$ methanol lines at $\simeq 96.74 \mathrm{GHz}$ toward the methanol emission peak near L1544 (J2000 $\alpha=05^{\mathrm{h}} 04^{\mathrm{m}} 18^{\mathrm{s}} \cdot 0$, $\delta=+25^{\circ} 11^{\prime} 10^{\prime \prime}$, Bizzocchi et al. 2014) were carried out with the NOEMA interferometer in C and D configurations on 2015 July 21-23 and 30 and October 25-26 under average weather conditions ( $\mathrm{pwv}=1-10 \mathrm{~mm}$ ). The rest frequencies are given in Table 1 . The primary beam size was $52^{\prime \prime}$, the synthesized beam was 5 !" $71 \times 3$ " 86 at a position angle $\theta=-52^{\circ} .48$. The data were obtained with the narrowband correlator with a spectral resolution of $39 \mathrm{kHz}$, corresponding to a velocity resolution of $0.12 \mathrm{~km} \mathrm{~s}^{-1}$. The system temperatures were 70-250 K. Sources $0234+285$, MWC349, LKHA101, and $0507+179$ were used as flux calibrators; $0507+179$ was 
Table 1

The Observed Methanol Lines

\begin{tabular}{lcccc}
\hline \hline Transition & $\begin{array}{c}\text { Frequency }^{\mathrm{a}} \\
(\mathrm{GHz})\end{array}$ & $\begin{array}{c}E_{\mathrm{up}} / k^{\mathrm{a}} \\
(\mathrm{K})\end{array}$ & $\begin{array}{c}A^{\mathrm{a}} \\
\left(10^{-5} \mathrm{~s}^{-1}\right)\end{array}$ & $\begin{array}{c}n_{\text {crit }}^{\mathrm{b}} \\
\left(10^{5} \mathrm{~cm}^{-3}\right)\end{array}$ \\
\hline$\left(2_{1,2}-1_{1,1}\right)-E_{2}$ & 96.739362 & $12.53^{\mathrm{c}}$ & 0.2558 & 0.82 \\
$\left(2_{0,2}-1_{0,1}\right)-A^{+}$ & 96.741375 & 6.96 & 0.3408 & 1.09 \\
$\left(2_{0,2}-1_{0,1}\right)-E_{1}$ & 96.744550 & $20.08^{\mathrm{c}}$ & 0.3407 & 1.09 \\
\hline
\end{tabular}

Notes.

a The frequencies, energies, and Einstein coefficients are taken from Bizzocchi et al. (2014), following Xu \& Lovas (1997) and Lees \& Baker (1968), also available at the JPL database (Pickett et al. 1998).

$\mathrm{b}$ The critical densities are calculated for a kinetic temperature of $10 \mathrm{~K}$.

${ }^{\mathrm{c}}$ Energy relative to the ground $0_{0,0}$, A rotational state.

used as a phase/amplitude calibrator; and 3C454.3 and 3C84 were used as bandpass calibrators.

Simultaneously, the dimethyl ether $\left(\mathrm{CH}_{3} \mathrm{OCH}_{3}\left(5_{5,1}-4_{4,0}\right)-E A\right.$ at $95.85 \mathrm{GHz})$ and methyl formate $\left(\mathrm{CH}_{3} \mathrm{OCHO}\left(5_{4,1}-5_{3,3}\right)-E\right.$ at $96.94 \mathrm{GHz}$ and $\left(17_{5,12}-17_{4,13}\right)-A$ at $\left.97.20 \mathrm{GHz}\right)$ lines were observed with the same spectral setup with the narrowband correlator but were not detected (the rms was $6 \mathrm{mJy}_{\text {beam }}{ }^{-1}$ with the synthesized beam size of 5 ! $7 \times 3$ !"9). Dust continuum emission observed with the wide band correlator WideX was not detected down to an rms noise level of $0.026 \mathrm{mJy}^{\text {beam }}{ }^{-1}$ with a beam size of 3 !" $4 \times 2$ !" 4 . The spectral range of WideX was $95.85-99.45 \mathrm{GHz}$. The $\mathrm{SO}\left(2_{3}-1_{2}\right)$ line at $99.30 \mathrm{GHz}$ and the $\mathrm{CS}(2-1)$ line at $97.98 \mathrm{GHz}$ lines were detected in the WideX band with a spectral resolution of $1950 \mathrm{kHz}$ or 5.9 and $6.0 \mathrm{~km} \mathrm{~s}^{-1}$ at the given frequencies, respectively. The peak intensities were $\sim 4.0 \mathrm{mJy} \mathrm{beam}^{-1}$ and $\sim 3.5 \mathrm{mJy}$ beam $^{-1}$ for CS and SO, respectively, and the rms was

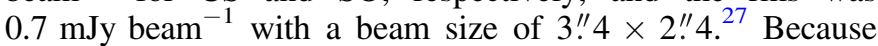
of their poor spectral resolution compared to the methanol lines, these data will not be discussed in this paper.

To recover the emission from scales larger than $20^{\prime \prime}$, we combined the synthetic visibilities derived from the IRAM $30 \mathrm{~m}$ observations of the methanol lines obtained by Bizzocchi et al. (2014) with our NOEMA data. The single-dish observations were carried out in 2013 October under excellent weather conditions (pwv $\simeq 0.5 \mathrm{~mm})$. The on-the-fly maps were obtained with the EMIR 090 (3 mm band) heterodyne receiver in position switching mode, using the FTS backend with a spectral resolution of $50 \mathrm{kHz}$; this corresponds to a velocity resolution of $0.15 \mathrm{~km} \mathrm{~s}^{-1}$ at the frequency of $96.74 \mathrm{GHz}$. The angular resolution was 25". 6 . The $3^{\prime} \times 3^{\prime}$ maps were centered at the dust emission peak (J2000 $\left.\alpha=05^{\mathrm{h}} 04^{\mathrm{m}} 17^{\mathrm{s}} .21, \delta=+25^{\circ} 10^{\prime} 42^{\prime \prime} \cdot 8\right)$. The pointing accuracy of the $30 \mathrm{~m}$ antenna was better than $1^{\prime \prime}$. The system temperature was $\simeq 90 \mathrm{~K}$ (for details, see Bizzocchi et al. 2014).

\subsection{Data Reduction: Spectral Data Cubes}

The calibration, imaging, and cleaning of the NOEMA data were performed with the CLIC and MAPPING packages of the GILDAS software. ${ }^{28}$ The single-dish data reduction up to the stage of convolved spectral data cubes was performed with

\footnotetext{
27 The sizes of the synthesized beams for methanol and other lines observed with the narrowband correlator and for continuum and the lines observed with WideX are different because, for the narrowband correlator observations, six antennas are used, while, for WideX observations, seven to eight antennas are used. The number of antennas used impacts the uv coverage and the synthesized beam size.

${ }^{28}$ The GILDAS software is developed at the IRAM and the Observatoire de Grenoble, and is available at http://www.iram.fr/IRAMFR/GILDAS.
}

the CLASS package of GILDAS. The comparison of the peak intensities of the methanol lines observed with the NOEMA and with the $30 \mathrm{~m}$ antenna shows that the interferometric observations recover $50 \%-60 \%$ of the total flux. To recover the missing flux we merged the NOEMA and the $30 \mathrm{~m}$ data with a standard routine in the MAPPING package. The resulting data cubes have a velocity resolution of $0.15 \mathrm{~km} \mathrm{~s}^{-1}$, the same as the single-dish data. After the correction for the NOEMA primary beam response function, the rms of the resulting spectral data cubes varies from 0.003 in the center to $0.009 \mathrm{Jy} \mathrm{beam}^{-1}$ at the edges of the primary beam. The synthesized beam of the combined data cube is 6 ." $50 \times 4$." 06 at a position angle $\theta=-49^{\circ} .95$, with a pixel size $1 . .5 \times 1$." 5 .

\subsection{Pyspeckit Line Fitting}

The line fitting was performed with the Pyspeckit module of Python (Ginsburg \& Mirocha 2011). The three methanol lines were fitted with a Gaussian profile in each pixel. The routine varies three parameters (peak intensity, centroid velocity $V_{\mathrm{LSR}}$, and velocity dispersion $\sigma$ ) and finds the best fit with the Levenberg-Marquardt nonlinear regression algorithm. The velocity dispersion was corrected for the channel width. The fit results were written to the final data cubes after masking poor data. In particular, for the integrated intensity maps, we used all data within the primary beam. For the centroid velocity and velocity dispersion we used the data within the primary beam, with a velocity dispersion accuracy better than $20 \%(\sigma / \Delta \sigma>5)$, and with a high signal-to-noise ratio $(\mathrm{S} / \mathrm{N}): I>5 \cdot \mathrm{rms} \cdot \sqrt{N_{\mathrm{ch}}} \cdot \Delta v_{\text {res }}$, where $I$ is the integrated intensity, $N_{\mathrm{ch}}$ is the number of channels in the line, and $\Delta v_{\text {res }}$ is the velocity resolution. For $N_{\mathrm{ch}}$, we take all channels in the range $6.1-8.0 \mathrm{~km} \mathrm{~s}^{-1}$. This range defines the emission above one $\mathrm{rms} \cdot \sqrt{N_{\mathrm{ch}}} \cdot \Delta v_{\text {res }}$ over the spectrum averaged over the whole mapped area.

\section{Results}

\subsection{Distribution of Methanol Emission}

Figure 2 shows the integrated intensity of the $E_{2}$ methanol line before combining the NOEMA data with the zero-spacing data from the $30 \mathrm{~m}$ antenna. Here, only the compact emission resolved by NOEMA is present. The methanol emission detected with NOEMA has an elongated structure on the northern side of the dense core in a direction that is about perpendicular to the direction of the main axis of the dense core and to the structure seen in the NOEMA $+30 \mathrm{~m}$ map (shown in Figure 3); its thickness is about $10^{\prime \prime}$ (1400 au at a distance of $140 \mathrm{pc}$ ) and the structure partly overlaps with the millimeter dust emission, from the $3 \sigma_{S_{1.2 \mathrm{~mm}}}$ up to the $6 \sigma_{S_{1.2 \mathrm{~mm}}}$ contour. The substructure might be due to a local abundance variation; the substructure orientation resembles the direction of the northern filament in the large-scale dust continuum emission map displayed in Figure 13 and it could be tracing the "contact point" between the two filaments.

The compact emission disappears partially after combining NOEMA with $30 \mathrm{~m}$ data, as shown in Figure 3 for the brightest methanol line $A^{+}$(the integrated intensity maps of the $E_{1}$ and $E_{2}$ lines are shown in the Appendix; see Figure 15). This map clearly shows a ridge elongated in approximately the same direction as the main axis of the dense core, with a thickness of $\sim 3000 \mathrm{au}$; the ridge contains a well defined peak at $\alpha=05^{\mathrm{h}} 04^{\mathrm{m}} 17^{\mathrm{s}} .68, \quad \delta=+25^{\circ} 11^{\prime} 08^{\prime \prime} .3$, with a size of 


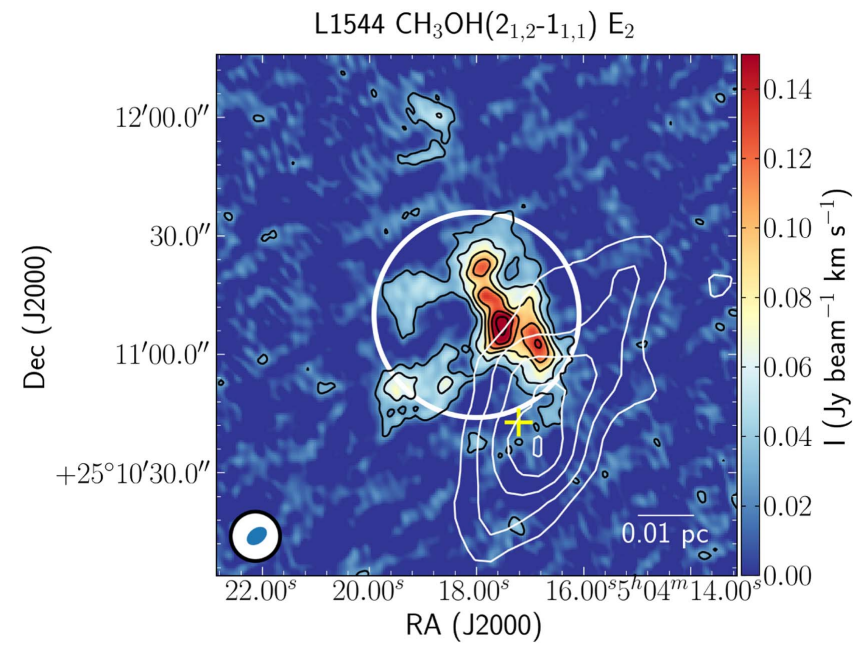

Figure 2. Integrated intensity of the $E_{2}$ line before combining with the singledish data (the map is not corrected for the primary beam attenuation). The black contours start at $3 \sigma_{S}\left(0.027 \mathrm{Jy}_{\text {beam }}^{-1}\right)$ with a step of $3 \sigma_{S}$. The white contours represent the $1.2 \mathrm{~mm}$ dust continuum emission from NIKA (ChacónTanarro et al. 2017). They start at $3 \sigma_{S}\left(0.021 \mathrm{Jy} \mathrm{beam}^{-1}\right.$, with the beam size of $12 . " 5)$ and increase with a step of $1 \sigma_{S}$. The white circle in the center is the primary beam of NOEMA for the methanol data. The yellow cross shows the dust emission peak (Ward-Thompson et al. 1999). The synthesized beam of NOEMA (blue) and the NIKA beam (white) are shown in the bottom left corner.

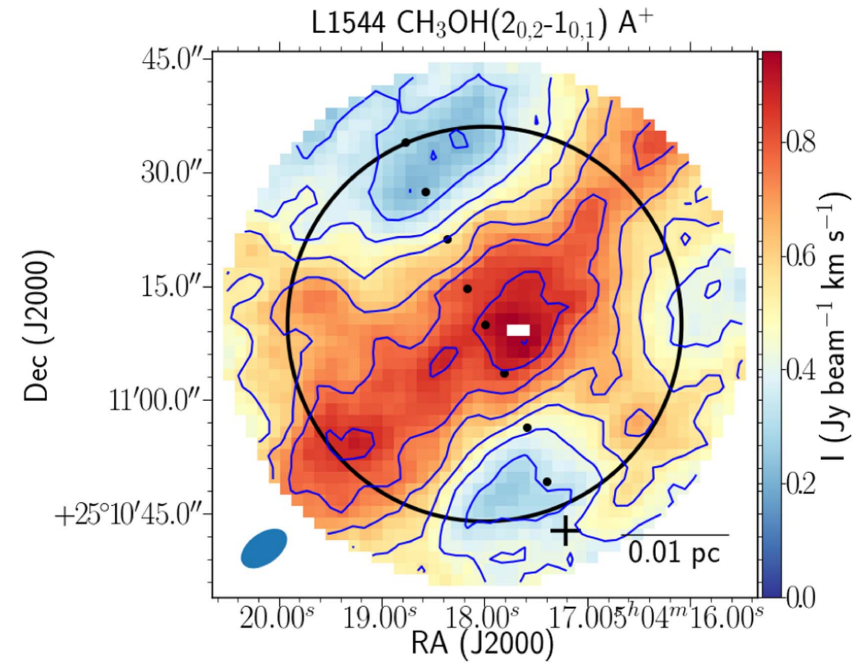

Figure 3. Integrated intensity of the $A^{+}$methanol line (NOEMA $\left.+30 \mathrm{~m}\right)$. The blue contours represent integrated intensity, and start at $0.216 \mathrm{Jy} \mathrm{beam}^{-1} \mathrm{~km} \mathrm{~s}^{-1}$ with a step of $0.108 \mathrm{Jy} \mathrm{beam}^{-1} \mathrm{~km} \mathrm{~s}^{-1}$. $3 \sigma_{I}=0.005 \mathrm{Jy}_{\text {beam }}^{-1} \mathrm{~km} \mathrm{~s}^{-1}$. The white circle is the NOEMA primary beam. The black cross shows the dust emission peak (Ward-Thompson et al. 1999). The synthesized beam of NOEMA is shown in the bottom left corner. The black dots show the positions of spectra used for non-LTE modeling (see Section 3.5 for details). The white pixels are those masked because of low-quality spectra.

$\sim 1300 \mathrm{au}$, and two secondary peaks southeast of the main peak. This ridge could be the zone where the two filaments are interacting and/or where cloud material is accreting onto the dense core. The map is centered at the methanol emission peak revealed with the IRAM $30 \mathrm{~m}$ observations (Bizzocchi et al. 2014). The dust emission peak at $1.3 \mathrm{~mm}$ from WardThompson et al. (1999) (considered as the core center) is shown with a black cross in Figure 3, outside the primary beam area. Chemical models of spherically symmetric prestellar

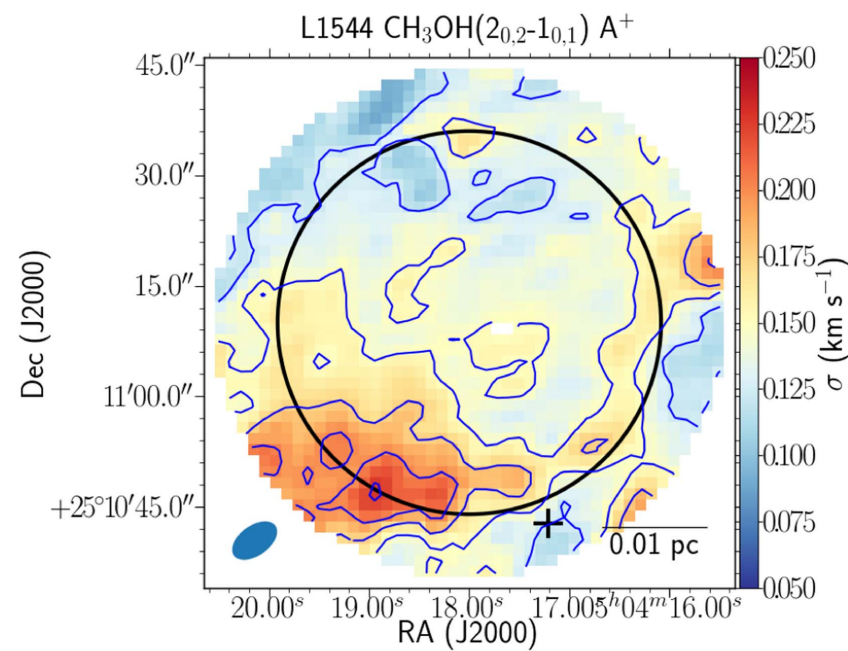

Figure 4. Velocity dispersions of the $A^{+}$methanol line. The blue contours show velocity dispersions of $0.125,0.150,0.175,0.200$, and $0.225 \mathrm{~km} \mathrm{~s}^{-1}$. The black circle shows the primary beam. The cross shows the $1.3 \mathrm{~mm}$ dust emission peak (Ward-Thompson et al. 1999). The synthesized beam is shown in the bottom left corner. The white pixels are those masked because of lowquality spectra.

cores predict that gas-phase methanol should be found in a shell around the dense core central regions (e.g., Vasyunin et al. 2017), giving rise to a ring-like structure in observations (e.g., Tafalla et al. 2006). Although the methanol emission is distributed around the dust continuum peak of L1544 (see Bizzocchi et al. 2014, and Figure 1), the ring-like structure is not uniform, with a clear maximum about 4000 au to the northeast of the dust peak position. The asymmetric distribution of methanol could be related to the inhomogeneities in the distribution of cloud material around the dense core, with the southern part more exposed to the interstellar radiation field (Spezzano et al. 2016). Gas-phase methanol preferentially traces the more shielded material around the dense core, where carbon is mainly locked in $\mathrm{CO}$ molecules.

\subsection{Kinematics}

\subsubsection{Velocity Dispersion}

Figures 4 and 16 show the velocity dispersions ( $\sigma=\Delta v / \sqrt{8 \ln (2)}$, where $\Delta v$ is the full width at half maximum, FWHM) of the methanol lines. The velocity dispersions range from 0.11 to $0.26 \mathrm{~km} \mathrm{~s}^{-1}$ with a median value of $0.15 \mathrm{~km} \mathrm{~s}^{-1}$ and typical uncertainties of $0.008 \mathrm{~km} \mathrm{~s}^{-1}$ and $0.014 \mathrm{~km} \mathrm{~s}^{-1}$ for the bright lines $\left(A^{+}\right.$and $\left.E_{2}\right)$ and weak line $\left(E_{1}\right)$. The line width increases toward the southeast clearly in the $A^{+}$and $E_{2}$ lines, and tentatively also in the $E_{1}$ line.

Toward the southeast, the velocity dispersions in the $(\mathrm{NOEMA}+30 \mathrm{~m})$ combined map are larger than those observed with the $30 \mathrm{~m}$ single-dish telescope by $0.05-0.10 \mathrm{~km} \mathrm{~s}^{-1}$. This difference is not systematic: it decreases with distance from the location with the largest dispersion in the southeast of the core and becomes negligible in the northern part of the map. A detailed inspection of the spectra reveals the presence of a small-scale higher velocity part of the line in the southeastern part of the core. NOEMA, being more sensitive than the $30 \mathrm{~m}$ antenna, reveals a weak higher velocity component, associated with the small-scale structure seen by NOEMA-only (see Figure 2), too small to be detected with the large beam of the single dish. In the combined spectrum, the resulting line is 


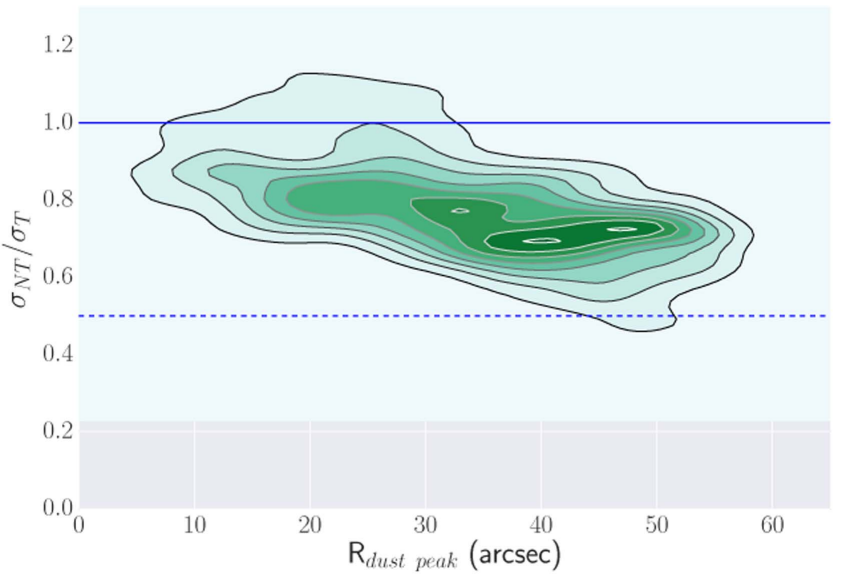

Figure 5. Ratio of nonthermal components of the three methanol lines to the thermal line width of a mean particle as a function of distance from the dust peak. The solid and dashed blue horizontal lines show the $\sigma_{\mathrm{NT}} / \sigma_{T}$ ratios equal to 1 and 0.5 . The colorscale and gray contours represent the number of the data points. Only the data points within the primary beam are used for the plot. The gray area does not contain any data points.

slightly broader and its centroid velocity is slightly larger than that observed with the single dish. The high-velocity part of the line detected with NOEMA appears toward the southern part of the primary beam, closer to the densest regions of the prestellar core.

\subsubsection{Nonthermal Motions}

Figure 5 shows the ratio of the nonthermal components $\left(\sigma_{\mathrm{NT}}\right)$ of the three methanol lines in each pixel within the primary beam and the thermal velocity dispersion of a mean particle, $\sigma_{T}$, as a function of the distance to the dust peak. The nonthermal components are derived from the observed velocity dispersion $\left(\sigma_{\mathrm{obs}}\right)$ via

$$
\sigma_{\mathrm{NT}}^{2}=\sigma_{\mathrm{obs}}^{2}-\frac{k T_{k}}{m_{\mathrm{obs}}}
$$

where $k$ is Boltzmann's constant, $T_{k}$ is the kinetic temperature, and $m_{\mathrm{obs}}$ is the mass of the observed molecule. The formula is adopted from Myers et al. (1991). The thermal velocity dispersion of a mean particle is $\sigma_{T}=\sqrt{k T_{k} / \mu}$, where $\mu=2.37 \mathrm{amu}$ is the mean particle mass (Kauffmann et al. 2008). We assume that the kinetic temperature is $10 \mathrm{~K}$ as this is the temperature measured with ammonia by Crapsi et al. (2007) at the distance of the methanol peak. This temperature is also consistent with the methanol rotational temperatures toward the high density gas close to the dust peak, where methanol is close to local thermodynamic equilibrium (LTE; see Section 3.3 for details).

The thermal velocity dispersion $\left(\sigma_{T}\right)$ for a mean particle with mass $2.37 \mathrm{amu}$ at $10 \mathrm{~K}$ is $0.19 \mathrm{~km} \mathrm{~s}^{-1}$. The ratio of the nonthermal component to the thermal velocity dispersion varies from 0.3 to 1.7 , being 0.8 on average. The ratio decreases with distance from the dust peak from $\sim 0.9$ to $\sim 0.7$ and it reaches unity toward the southeast. The majority of the lines (92\%) are subsonic, with the small fraction of transonic lines coming from the southeast region.

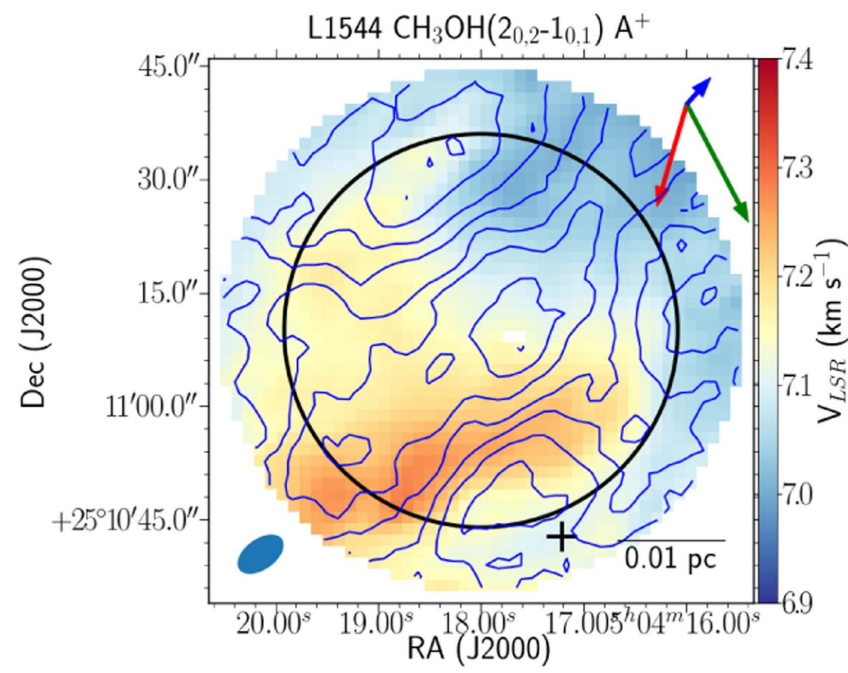

Figure 6. Centroid velocities of the $A^{+}$methanol line. The blue contours represent the integrated intensity, starting at $0.216 \mathrm{Jy} \mathrm{beam}^{-1} \mathrm{~km} \mathrm{~s}^{-1}$ with a step of $0.108 \mathrm{Jy} \mathrm{beam}^{-1} \mathrm{~km} \mathrm{~s}^{-1} ; 3 \sigma_{I}=0.005 \mathrm{Jy} \mathrm{beam}^{-1} \mathrm{~km} \mathrm{~s}^{-1}$. The red arrow shows the total velocity gradient measured with the methanol line $\left(7.10 \pm 0.01 \mathrm{~km} \mathrm{~s}^{-1} \mathrm{pc}^{-1}\right)$. The green and blue arrows show the total velocity gradients of $\mathrm{NH}_{3}$ and $\mathrm{NH}_{2} \mathrm{D}$ measured at scales of 15,000 au and $4000 \mathrm{au}$, respectively (Crapsi et al. 2007; the length of the arrow is proportional to the velocity gradient magnitude). The circle shows the primary beam. The black cross shows the $1.3 \mathrm{~mm}$ dust emission peak (Ward-Thompson et al. 1999). The synthesized beam is shown in the bottom left corner. The white pixels are those masked because of low-quality spectra.

\subsubsection{Velocity Field}

Figures 6 and 17 show the centroid velocity $\left(V_{\mathrm{LSR}}\right)$ maps. The $V_{\mathrm{LSR}}$ varies in the range $6.9-7.3 \mathrm{~km} \mathrm{~s}^{-1}$. The interferometric observations reveal substructure in the velocity field, with the velocity increasing toward the south, southeast, and east. The three lines show similar velocity patterns; the $E_{1}$ line shows higher velocities in the southeast, where the $\mathrm{S} / \mathrm{N}$ of the weak $E_{1}$ line is low $(\simeq 5)$ compared to the $E_{2}$ and $A^{+}$lines; $\mathrm{S} / \mathrm{Ns}$ there are $>20$. We estimate total and local velocity gradients across the methanol emission following the method described in Goodman et al. (1993) for total gradients and applied for local gradients by Caselli et al. (2002a; see the description of local gradients below). The total velocity gradient calculation provides the average velocity across the mapped region, $\left\langle V_{\mathrm{LSR}}\right\rangle$, the magnitude of the velocity gradient, $G$, and the position angle, $\theta_{G}$. The total gradients are calculated using all available points weighted by $1 / \Delta_{V_{\mathrm{LSR}}}^{2}$, where $\Delta_{V_{\mathrm{LSR}}}$ is the uncertainty of the centroid velocity.

The total gradients have been measured for the three methanol lines: $G=8.77 \pm 0.04,7.10 \pm 0.01$, and $7.24 \pm$ $0.01 \mathrm{~km} \mathrm{~s}^{-1} \mathrm{pc}^{-1}$, with $\theta_{G}=153^{\circ} .7 \pm 0.3,162^{\circ} .13 \pm 0^{\circ} .08$, and $163^{\circ} .76 \pm 0.07$ measured east of north, for the $E_{1}, A^{+}$, and $E_{2}$ lines, respectively. The total gradient for the $A^{+}$line is shown as red arrows in Figures 6 and 7. For comparison, the green and blue arrows in Figure 6 represent the velocity gradients measured with the high density tracers $\mathrm{NH}_{3}$ and $\mathrm{NH}_{2} \mathrm{D}$ using interferometric data across the entire L1544 core (Crapsi et al. 2007; with the arrow size proportional to the magnitude of the velocity gradient). The $\mathrm{NH}_{3}$ traces the whole dense core (radius $\sim 15,000 \mathrm{au}$ ), while $\mathrm{NH}_{2} \mathrm{D}$ traces the inner region of the core, which includes the dust peak ( $\sim 4000 \mathrm{au}$; see Crapsi et al. 2007, for details). Williams et al. (2006) also measure the total gradient of the dense core with the interferometric observations of $\mathrm{N}_{2} \mathrm{H}^{+}\left(G=4.1 \mathrm{~km} \mathrm{~s}^{-1} \mathrm{pc}^{-1}\right)$. 


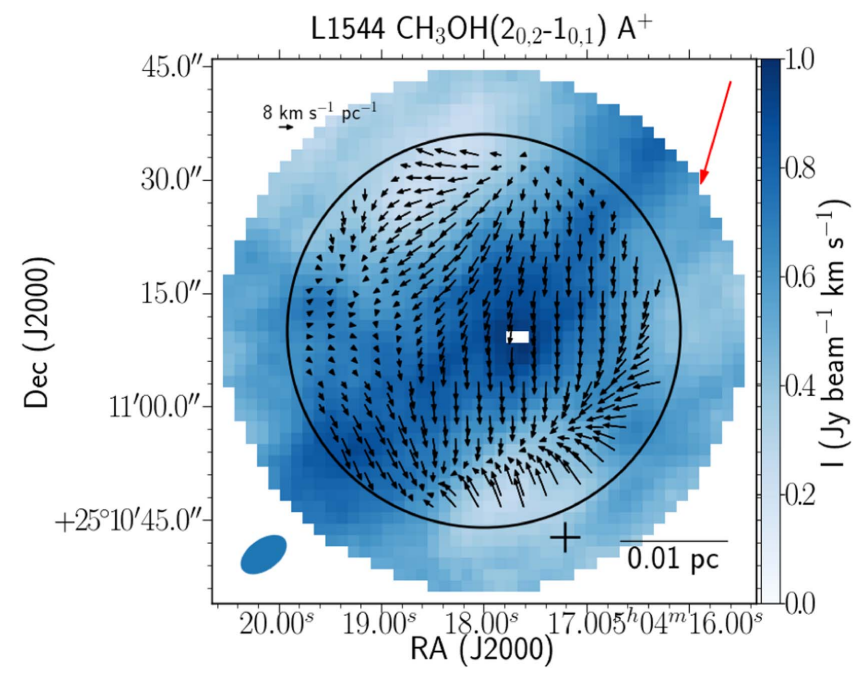

Figure 7. Local velocity gradients of the $A^{+}$methanol line. The colorscale shows integrated intensity. The black arrows indicate the local velocity gradients. The red arrow shows the total velocity gradient (the scale of the total gradient is eight times larger than the scale of the local gradients). The circle shows the primary beam. The cross denotes the $1.3 \mathrm{~mm}$ dust emission peak (Ward-Thompson et al. 1999). The synthesized beam is plotted in the bottom left corner. The white pixels are those masked because of low-quality spectra.

The gradient direction found using methanol significantly differs from those of the dense core tracers $\left(\theta_{G}\right.$ differs by $\sim 20^{\circ}$, $\sim 40^{\circ}$, and $\sim 140^{\circ}$ from those of $\mathrm{N}_{2} \mathrm{H}^{+}, \mathrm{NH}_{3}$, and $\mathrm{NH}_{2} \mathrm{D}$, respectively) so we can conclude that the shell traced by methanol is not following the dense core kinematics. It is also interesting to compare the total velocity gradient deduced by methanol to the direction of the large-scale magnetic field measured in the northern part of L1544 $\left(\theta_{B}=30^{\circ}\right.$; Clemens et al. 2016). The minimum difference between the total velocity gradient direction at the scale of the NOEMA methanol map and the magnetic field direction is $\simeq 50^{\circ}$, thus showing gas motions not aligned with larger scale magnetic field directions; this may indicate changes in the magnetic field direction toward the dense regions of the prestellar core as also pointed out by Clemens et al. (2016).

The local velocity gradients are presented in Figures 7 and 18 with black arrows, plotted over integrated intensity color maps, along with red arrows that present the total velocity gradients. To calculate a gradient in a local position, we use all pixels within $6^{\prime \prime}$ (where 1 pixel is $1 . ! 5$ in size), weighted according to their distance to the given position and their centroid velocity uncertainty:

$$
w=\frac{1}{\Delta_{V_{\mathrm{LSR}}}^{2}} \cdot \exp \left\{-d^{2} /\left[2\left(\frac{\theta_{\mathrm{Gauss}}}{2.354}\right)^{2}\right]\right\}
$$

where $w$ is the weight, $\Delta_{V_{\text {LSR }}}$ is the centroid velocity uncertainty, $d$ is the distance from the weighted pixel to the given position, and $\theta_{\text {Gauss }}=4$ pixels is the FWHM of the weighting function. The four-pixel radius is used to compensate for the oversampling of the map. The typical errors for the local gradient values are $1 \mathrm{~km} \mathrm{~s}^{-1} \mathrm{pc}^{-1}$ for the $E_{1}$ line and $0.2 \mathrm{~km} \mathrm{~s}^{-1} \mathrm{pc}^{-1}$ for the $E_{2}$ and $A^{+}$lines; and for the position angles of the local gradients $-4^{\circ}$ for the $E_{1}$ line and 1.5 for the $E_{2}$ and $A^{+}$lines.
The velocity increases toward the southeast, as shown with the total velocity gradient direction. However, the arrows now indicate that the velocity field is not smooth and is quite complex, with local velocity gradients showing significant variations in magnitude and direction across the observed area. The local velocity gradient values vary from $\simeq 0.5$ to $12 \mathrm{~km} \mathrm{~s}^{-1} \mathrm{pc}^{-1}$. There is a high-velocity bar in the south of the mapped area and then a sharp decrease (local velocity gradients are $\simeq 11 \mathrm{~km} \mathrm{~s}^{-1} \mathrm{pc}^{-1}$ ) of the centroid velocity further to the south, toward the dust peak (see Figures 6 and 7). This sudden inversion of the direction in velocity gradient could be reproduced at the intersection of flows moving in different directions along the line of sight, which could support the scenario of the gentle collision between the two large-scale filaments clearly seen in the Herschel map shown in Figure 13. However, one cannot exclude complex kinematics due to accretion of material at the northeastern edge of the quiescent prestellar core.

\subsection{Rotational Temperature}

Using the spectra of the observed lines, we calculate the rotational temperature $T_{\text {rot }}$ and the total column density $N_{\text {tot }}$ of methanol, assuming LTE and optically thin emission. We assume the $A: E$ methanol ratio to be $1: 1$. With the assumption of LTE, the population of all the energy levels can be described by a unique temperature, $T_{\text {rot }}$. With the assumption of optically thin emission, $T_{\text {rot }}$ is defined as $-1 / a$ from a linear fit $a x+b$ to a $\log \left(N_{\text {up }} / g_{\text {up }}\right)$ versus $E_{\text {up }}$ plot (rotational diagram), where $E_{\text {up }}$ is the energy of the upper level, expressed in $\mathrm{K}$ (given in Table 1); $N_{\text {up }}$ is the column density of the upper level population, defined as

$$
N_{\text {up }}=\frac{8 \pi k W \nu^{2}}{A h c^{3}},
$$

where $k$ is the Boltzmann constant, $W$ is the integrated intensity of the line, $\nu$ is the frequency, $A$ is the Einstein coefficient (given in Table 1), $h$ is the Planck constant, and $c$ is the speed of light (e.g., Goldsmith \& Langer 1999).

Figure 8 shows the map of rotational temperature (left) and its uncertainty (right). We show only those values with an uncertainty $\Delta T_{\text {rot }}<2.5 \mathrm{~K}$. The typical values for $\Delta T_{\text {rot }}$ are $1-2 \mathrm{~K}$. $T_{\text {rot }}$ varies from $3.0 \pm 0.8 \mathrm{~K}$ to $9 \pm 2 \mathrm{~K}$, with an average $T_{\text {rot }}$ of $5.3 \pm 1.0 \mathrm{~K}$. The temperature increases toward the southwest and the dust peak. The rotational temperature increase is most likely a result of the gas volume density increase toward the core center, from a few $10^{4} \mathrm{~cm}^{-3}$ in the northeastern part of the observed area to a few $\times 10^{5} \mathrm{~cm}^{-3}$ in the southwestern part (see, e.g., the model of Keto \& Caselli 2010), as the methanol lines have critical densities of $\simeq 10^{5} \mathrm{~cm}^{-3}$. As the density increases toward the core center, the energy level populations become closer to those expected in LTE and the rotational temperature approaches the kinetic temperature of the gas.

Crapsi et al. (2007) derived ammonia rotational temperatures toward the core center and found that the temperature increases from the center outward, from 5.5 to $10-13 \mathrm{~K}$. The ammonia map obtained with the VLA covers the ammonia emission area of $75^{\prime \prime} \times 36^{\prime \prime}$ centered at the dust peak. The peak methanol rotational temperature of $9 \pm 2 \mathrm{~K}$ is consistent with these ammonia derived temperatures, suggesting that the methanol is indeed close to being in LTE in the southwestern part of the mapped region. It is also consistent with the kinetic temperature 

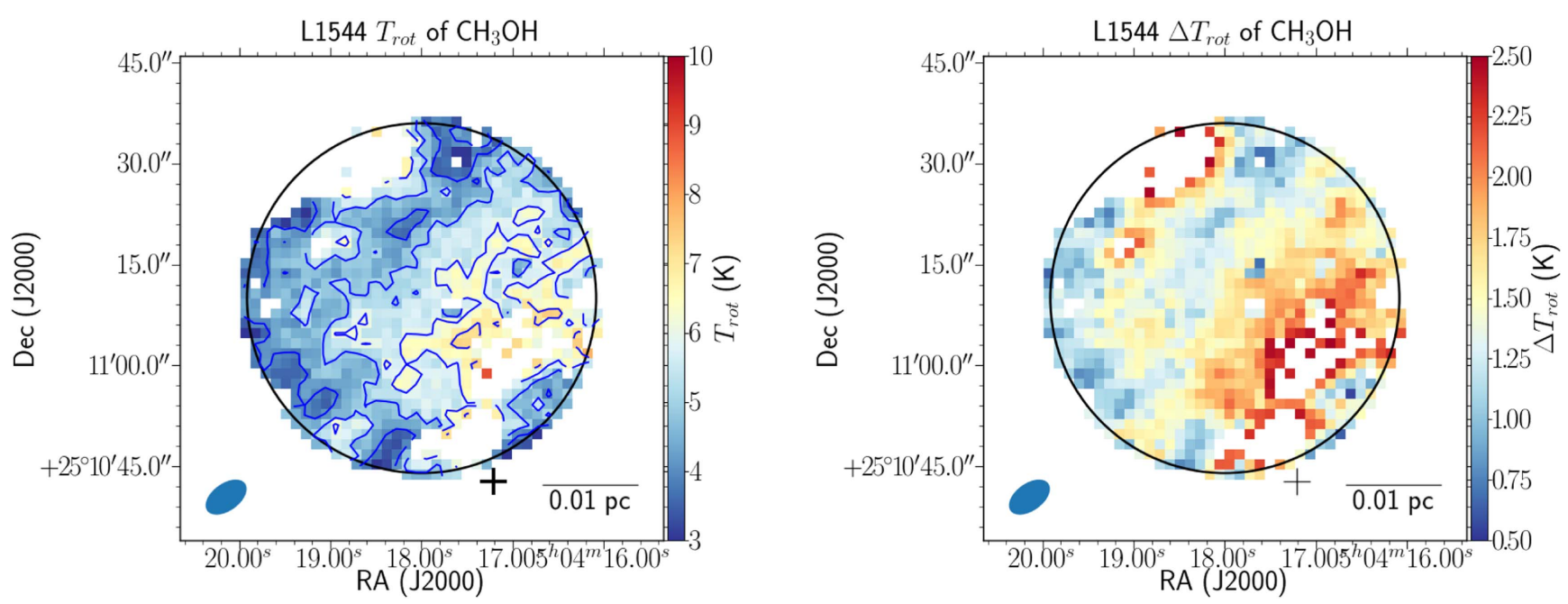

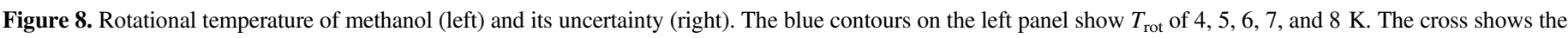

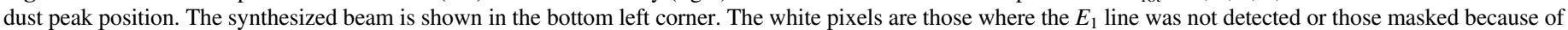
low $T_{\text {rot }}$ accuracy $\left(\Delta T_{\text {rot }} \geqslant 2.5 \mathrm{~K}\right)$.

derived with non-LTE modeling for methanol toward the L1544 dust peak by Vastel et al. (2014; 7-15 K). This result confirms that $\mathrm{CH}_{3} \mathrm{OH}$ is mainly tracing a shell around the L1544 dust peak, as toward the center, Crapsi et al. (2007) found temperatures of $\sim 6 \mathrm{~K}$ using $\mathrm{NH}_{3}$, which does not appear to freeze-out (see also Caselli et al. 2017). The opposing directions of the methanol and ammonia temperature gradients further support the notion that the methanol gradient is only an excitation effect caused by changes in the gas density. In general, $\mathrm{CH}_{3} \mathrm{OH}$ and $\mathrm{NH}_{3}$ trace different material, as $\mathrm{NH}_{3}$ (as well as other N-bearing molecules) remains in the gas phase at significantly higher volume densities compared to $\mathrm{C}$-bearing molecules (e.g., Caselli et al. 1999; Tafalla et al. 2002; HilyBlant et al. 2010; Bizzocchi et al. 2014), but $\mathrm{CH}_{3} \mathrm{OH}$ and $\mathrm{NH}_{3}$ overlap at densities between $10^{4}$ and $10^{5} \mathrm{~cm}^{-3}$, where $\mathrm{CH}_{3} \mathrm{OH}$ maintains a detectable abundance in the gas phase.

\subsection{Column Density and Methanol Abundance}

The total column density is given by

$$
N_{\text {tot }}=\frac{N_{\text {up }} Q_{\text {rot }}}{g \exp \left(-E_{\text {up }} / k T\right)},
$$

where $g$ is the statistical weight of the upper level $\left(g_{J}=2 J+1\right.$, with $J$ being the rotational quantum number), $E_{\text {up }}$ is the energy of the upper level, $k$ is the Boltzmann constant, $T_{\text {rot }}$ is used as the temperature $T$, and $Q_{\text {rot }}$ is the rotational partition function; its values for different temperatures are taken from the CDMS database (Müller et al. 2001).

Figure 9 shows the total column density map of methanol (left) and its uncertainty (right) derived for the brightest line, $A^{+}$. The variation between $N_{\text {tot }}$ found using the different lines is within a factor of 3: $N_{\text {tot }}\left(E_{2}\right) / N_{\text {tot }}\left(E_{1}\right) \simeq 2, N_{\text {tot }}\left(E_{2}\right) / N_{\text {tot }}\left(A^{+}\right) \simeq 3$. The uncertainties of the total column densities $\Delta N_{\text {tot }}$ are high: $30 \%$ $300 \%, 20 \%-100 \%$, and $20 \%-300 \%$ for the $E_{1}, A^{+}$, and $E_{2}$ lines, respectively, so the differences between the total column densities defined with the different lines are within the errors. The average column densities over the $30^{\prime \prime}$ beam close to the dust peak within the primary beam are $(3.0 \pm 0.9) \times 10^{13} \mathrm{~cm}^{-2}$, $(2.1 \pm 0.6) \times 10^{13} \mathrm{~cm}^{-2}$, and $(5.7 \pm 1.6) \times 10^{13} \mathrm{~cm}^{-2}$ for the $E_{1}, A^{+}$, and $E_{2}$ lines, respectively. This result is consistent with that of Bizzocchi et al. (2014), who found $(2.7 \pm 0.6) \times$ $10^{13} \mathrm{~cm}^{-2}$, and that of Vastel et al. (2014), who found (2.6-3.8) $\times 10^{13} \mathrm{~cm}^{-2}$, toward the dust peak observed with the IRAM $30 \mathrm{~m}$ telescope (beams of $30^{\prime \prime}$ and $26^{\prime \prime}$, thus also partially including the area mapped with NOEMA).

We use the $\mathrm{H}_{2}$ column density, $N\left(\mathrm{H}_{2}\right)$, to define the average methanol abundance within the primary beam area. The molecular hydrogen column density map for L1544 was produced by Spezzano et al. (2016) using the dust continuum emission data from the three Herschel/SPIRE bands at $250 \mu \mathrm{m}, 350 \mu \mathrm{m}$, and $500 \mu \mathrm{m}$. As the Herschel beam of $38^{\prime \prime}$ is comparable with the NOEMA primary beam at $96.4 \mathrm{GHz}$ $\left(52^{\prime \prime}\right)$, we can only define an average abundance of methanol within the observed area. With the average total column densities and molecular hydrogen column density (averaged over the $30^{\prime \prime}$ beam close to the dust peak within the NOEMA primary beam area), $(2.3 \pm 0.3) \times 10^{22} \mathrm{~cm}^{-2}$, the average methanol abundances

$$
X\left(\mathrm{CH}_{3} \mathrm{OH}\right)=N_{\text {tot }}\left(\mathrm{CH}_{3} \mathrm{OH}\right) / N\left(\mathrm{H}_{2}\right)
$$

are $(1.3 \pm 0.4) \times 10^{-9},(0.9 \pm 0.3) \times 10^{-9}$, and $(2.5 \pm 0.8) \times$ $10^{-9}$ for the $E_{1}, A^{+}$, and $E_{2}$ lines, respectively, consistent with $0.92 \times 10^{-9}$ found by Bizzocchi et al. (2014) and lower than that found by Vastel et al. (2014), $6 \times 10^{-9}$. The difference between our result and that of Vastel et al. (2014) is due to the lower molecular hydrogen column density they assumed $\left(5 \times 10^{21} \mathrm{~cm}^{-2}\right)$.

\subsection{Non-LTE Modeling}

To put better constraints on the column densities of methanol, taking into account the physical structure of the source, we perform non-LTE modeling of the methanol lines in the same manner as Bizzocchi et al. (2014). We use the radiative transfer code MOLLIE (Keto \& Rybicki 2010), which produces line synthetic spectra based on the physical model of L1544 derived by Keto et al. (2014; see Figure 10 for the density and temperature profiles). L1544 is modeled as an unstable slowly contracting Bonnor-Ebert sphere with radiative heating and cooling of the gas and dust and simplified $\mathrm{CO}$ and $\mathrm{H}_{2} \mathrm{O}$ chemistry to set the abundances of the major gas coolants 

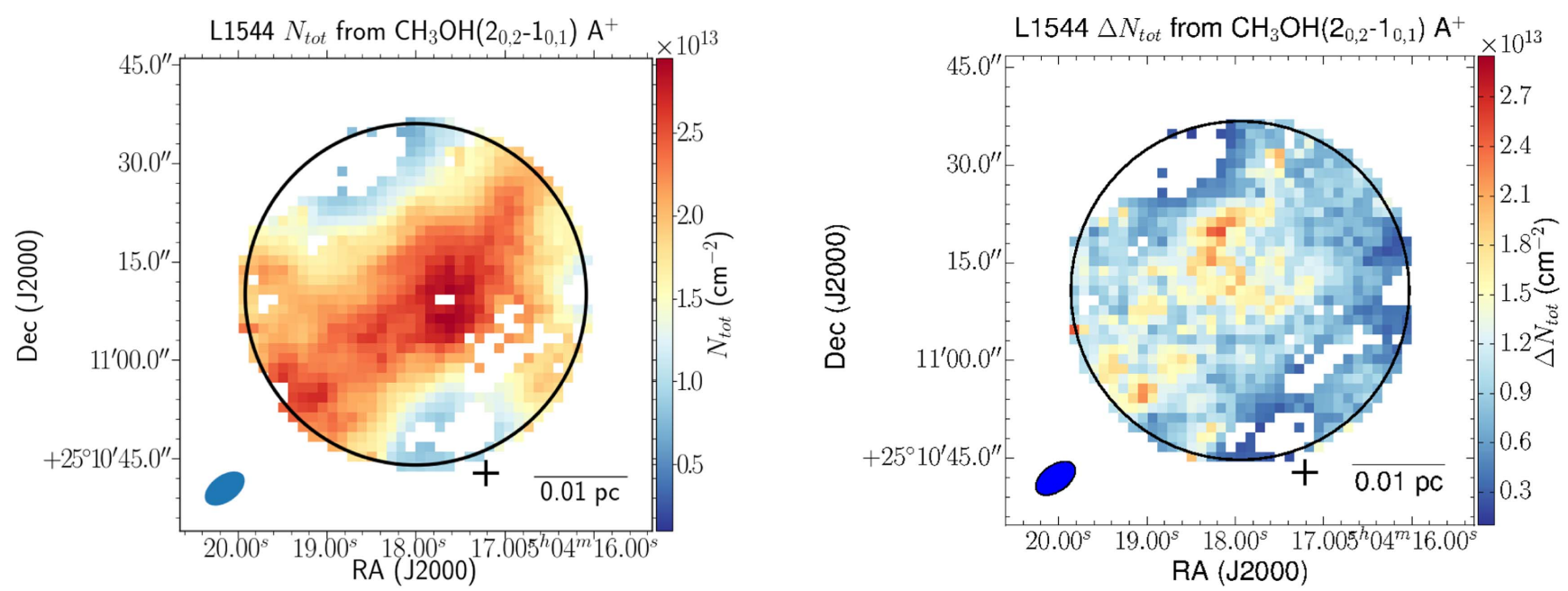

Figure 9. Total column densities of methanol measured with the $A^{+}$line (left) and its uncertainty (right) derived with the assumption of LTE. The circle shows the primary beam. The cross shows the dust peak position. The synthesized beam is shown in the bottom left corner. The white pixels are those where the $E_{1}$ line was not detected or those masked because of low $T_{\text {rot }}$ accuracy $\left(\Delta T_{\text {rot }} \geqslant 2.5 \mathrm{~K}\right)$.

(Keto \& Caselli 2008; Keto et al. 2014). The $\mathrm{CH}_{3} \mathrm{OH}$ abundance profile across the core is assumed to follow the abundance profile of the mother molecule, $\mathrm{CO}$, with $X\left(\mathrm{CH}_{3} \mathrm{OH}\right)=0.35 \times 10^{-9}$ toward the dust peak. We chose eight positions on the line connecting the dust peak and the methanol peak within the primary beam, so the distance between the positions is similar to the synthesized beam size (the positions are shown with black dots in Figure 3) to measure the methanol column densities along the core radius. Since methanol molecules do not switch their symmetries between the $A$ and $E$ forms, $\mathrm{CH}_{3} \mathrm{OH}-\mathrm{A}$ and $\mathrm{CH}_{3} \mathrm{OH}-E$ may be considered as two different molecules with similar abundances. We compare independently the observed methanol $A$ and $E$ spectra with modeled lines and find the abundance profile that better reproduces the observed lines. The modeled spectra are presented in Figure 11. The red lines represent the simulated spectra, after smoothing to the same velocity resolution as the observed spectra, which better resemble the observed spectra (black). The dark gray strips represent the modeled lines produced with one step smaller and one step larger column densities (one step is $10 \%$ of the given column density).

The resulting column density profiles (along the core radius through the methanol peak) are shown in Figure 12. The column densities of $\mathrm{CH}_{3} \mathrm{OH}-\mathrm{A}$ and $\mathrm{CH}_{3} \mathrm{OH}-E$ agree very well, within $10 \%$, which is consistent with the assumed 1:1 E:A abundance ratio. The modeled column density varies between $\simeq 0.7 \times 10^{13} \mathrm{~cm}^{-2}$ close to the dust peak and $\simeq 2.4 \times 10^{13} \mathrm{~cm}^{-2}$ toward the methanol peak for each $A$ - and $E$-methanol, that is, the total column density of methanol is $(1.4-4.8) \times 10^{13} \mathrm{~cm}^{-2}$. This result agrees very well with that calculated assuming LTE (see Section 3.4 and Figure 9). The low accuracies of the LTE- column densities allow large variations within the errors. However, the LTE column densities of the $A^{+}$line shown in Figure 9 present the same column density range $(0.7-3.0) \times 10^{13} \mathrm{~cm}^{-2}$ as the non-LTE profile (see Figure 12). The results of non-LTE modeling give a lower column density close to the dust peak ( $7^{\prime \prime}$ away from the dust peak) compared to the results of Bizzocchi et al. (2014) and Vastel et al. (2014): $N_{\text {tot }}\left(\mathrm{CH}_{3} \mathrm{OH}\right)=(2.7 \pm 0.6) \times 10^{13} \mathrm{~cm}^{-2}$ and $2.6-3.8 \times 10^{13}$ $\mathrm{cm}^{-2}$, respectively, while we derive $0.68 \times 10^{13} \mathrm{~cm}^{-2}$. This difference is likely due to the larger IRAM $30 \mathrm{~m}$ beam of 25 ". 5

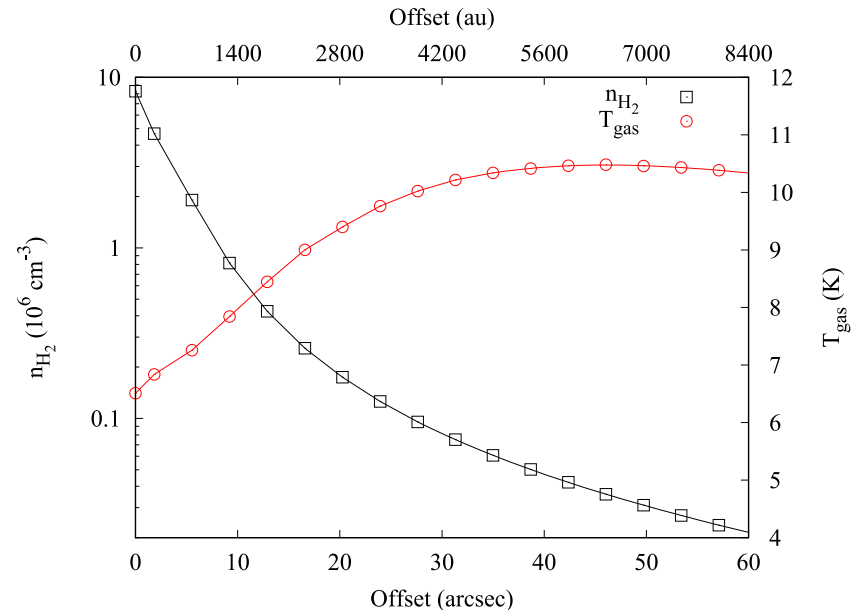

Figure 10. Physical structure of the modeled core: molecular hydrogen number density (black) and kinetic gas temperature (red).

used in both Bizzocchi et al. (2014) and Vastel et al. (2014) works, which dilutes the area where methanol is depleted.

\section{Discussion}

The single-dish observations of methanol toward L1544 (Bizzocchi et al. 2014) revealed an asymmetric ring-like structure of emission with a peak on the northeast side of the core. NOEMA has looked in detail at the $\mathrm{CH}_{3} \mathrm{OH}$ peak and found morphological substructure within the NOEMA primary beam, which cannot be resolved with the IRAM $30 \mathrm{~m}$ telescope. The interferometric observations have revealed a complex velocity structure. A clear velocity gradient with magnitude $\simeq 7 \mathrm{~km} \mathrm{~s}^{-1} \mathrm{pc}^{-1}$ is found and the velocity dispersion increases toward the southeast of the NOEMA image, reaching values larger by $0.05-0.1 \mathrm{~km} \mathrm{~s}^{-1}$ when compared to those measured with the single-dish. The higher velocity dispersion gas is concentrated toward the northeast edge of the prestellar core and may be produced by cloud material slowly accreting onto the core. Another possibility could be a transonic shock produced by the collision of the two almost perpendicular filaments seen in the large-scale Herschel dust continuum map (see Figure 13), with the L1544 methanol peak found at the 

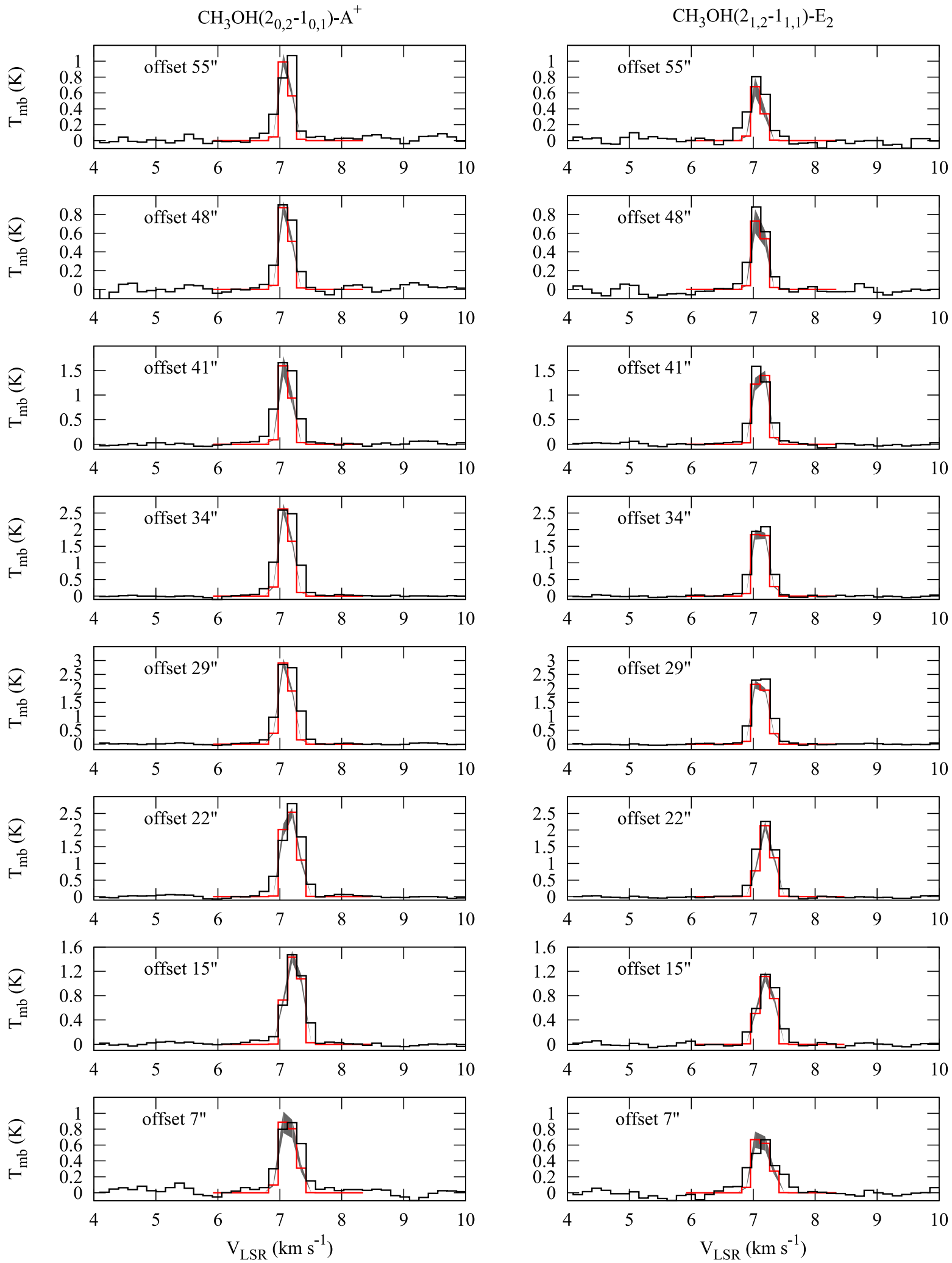

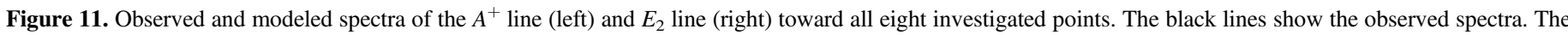
red lines show the best modeled lines. The gray strips show the lines modeled with the neighboring knots of the grid.

intersection of the two. The currently available methanol data, however, are not sufficient to identify conclusively the presence of a shock at this location, for example, with the finding of local gas temperature increase, as the temperature of the gas traced by $\mathrm{CH}_{3} \mathrm{OH}$ cannot be measured. In fact, the observed transitions have low energies (see Table 1) and thus are not sensitive to high temperatures, which are expected even in slow shocks (see, e.g., Pon et al. 2014). In the future, we plan to search for higher excitation lines of $\mathrm{CH}_{3} \mathrm{OH}$ and $\mathrm{CO}$ to investigate this further. 

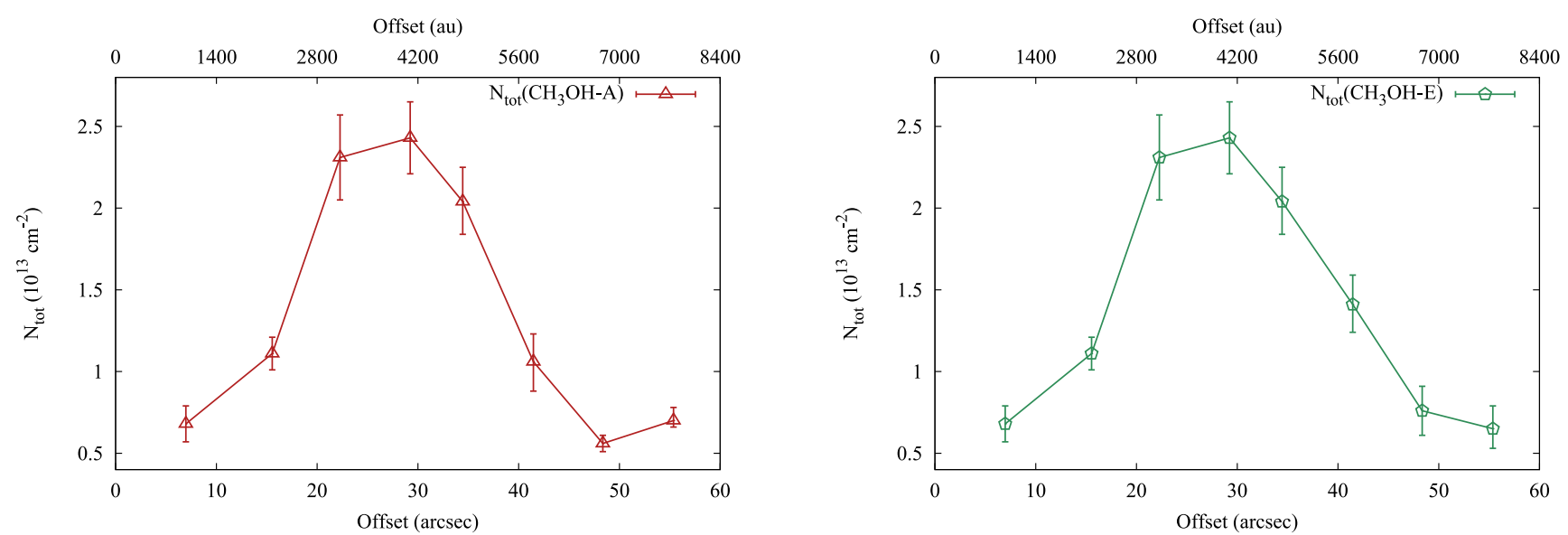

Figure 12. Modeled column densities of $\mathrm{CH}_{3} \mathrm{OH}-\mathrm{A}$ (left) and $\mathrm{CH}_{3} \mathrm{OH}-\mathrm{E}$ (right) toward eight selected positions across the core as a function of distance from the dust peak. The errorbars show the dispersion between the modeled column densities in the neighboring knots of the grid.

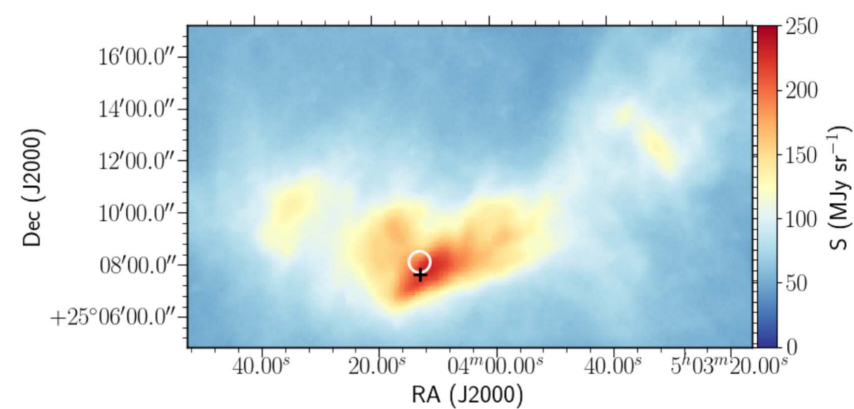

Figure 13. $250 \mu \mathrm{m}$ dust contimuum emission Herschel/SPIRE map toward the L1544 region (André et al. 2010). The white circle shows the NOEMA primary beam centered at the methanol peak. The black cross shows the $1.3 \mathrm{~mm}$ dust emission peak (Ward-Thompson et al. 1999).

The methanol column densities calculated with an assumption of LTE and determined with non-LTE modeling agree within the errors, but the column density close to the dust peak is two to three times smaller than that defined with the singledish observations by Bizzocchi et al. (2014) and Vastel et al. (2014). This can be explained by the large $30 \mathrm{~m}$ beam that partly dilutes the zone of depleted methanol. The rotational temperature calculations show that methanol is close to LTE only near the dust peak where it is significantly depleted. We compare the non-LTE molecular abundance profile to the modeled methanol abundance profile predicted with the MONACO code (Vasyunin et al. 2017), convolved to 5" beam, close to the size of the NOEMA beam (see Figure 14) and find one order of magnitude lower abundances than those predicted by the model. The shape of the derived abundance profile is consistent with the model predictions, although the model suggests a slight decrease in the abundance with the radius after the maximum at $\sim 7000 \mathrm{au}$, while the derived abundances start to decrease after the methanol peak at the radius of $\sim 4000$ au. This is considered to be a fair agreement given that the modeled abundance accuracy is one order of magnitude (Vasyunin et al. 2004, 2008), however, also suggests that the model overproduces the $\mathrm{CH}_{3} \mathrm{OH}$ possibly by overestimating the efficiency of reactive desorption, based on the laboratory results of Minissale et al. (2016a), or by overestimating the efficiency of atomic hydrogen tunneling through activation barriers of surface reactions taken from the work of Hasegawa et al. (1992). Another factor that can lead to the overproduction of methanol is that the static model does not

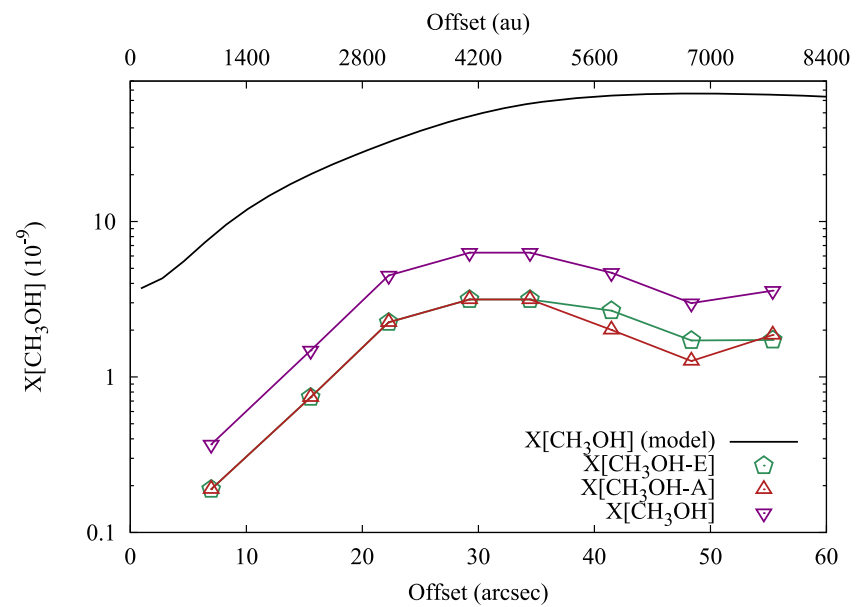

Figure 14. Abundances of $\mathrm{CH}_{3} \mathrm{OH}$ from non-LTE modeling with MOLLIE (A shown with red, E with green, and the total abundance, the sum of A-methanol and E-methanol abundances shown with purple) and the methanol abundances predicted by Vasyunin et al. (2017; black line).

allow dust temperature to exceed $10 \mathrm{~K}$, which prevents the formation of some major ice species such as $\mathrm{CO}_{2}$, where a significant fraction of carbon and oxygen may be locked. That is inclusion of physical evolution of L1544 in the model can lead to efficient formation of $\mathrm{CO}_{2}$ ice, and reduced abundances of other $\mathrm{C}$ - and $\mathrm{O}$-bearing species, including $\mathrm{CH}_{3} \mathrm{OH}$. It is also important to stress that the chemical model, as well as the radiative transfer analysis, assume spherical symmetry, and thus neglect the elongated structure and differential illumination described in Spezzano et al. (2016).

\section{Summary}

This paper presents high spatial resolution $(\sim 700$ au) observations of the methanol emission peak toward the prototypical prestellar core L1544 (revealed by Bizzocchi et al. 2014). The asymmetry of methanol emission around the dust peak of the prestellar core is likely caused by an irregular distribution of the core material and a lack of UV radiation at the methanol peak, as was suggested by Spezzano et al. (2016). NOEMA shows that the methanol peak has a smooth morphology, but reveals a complex velocity field. The increase in velocity dispersion toward the northeast edge of the prestellar core, where the local velocity 
gradients also present sharp changes in magnitude and direction, suggests that slow shocks are present. These slow shocks could be produced by either the accretion of cloud material onto the core or by a collision of the two filamentary structures seen in Herschel/ SPIRE images. The NOEMA observations, coupled with a nonLTE radiative transfer analysis, which takes into account the physical structure of the prestellar core and surroundings, also helped to unveil the methanol distribution along the line of sight; we deduced a (factor of 2-3) higher depletion of $\mathrm{CH}_{3} \mathrm{OH}$ close to the dust peak, when compared with results from single-dish observations. Comparison of the deduced $\mathrm{CH}_{3} \mathrm{OH}$ column densities with a chemical model applied to the L1544 physical structure suggests that the model is overpredicting the $\mathrm{CH}_{3} \mathrm{OH}$ abundance, probably because of a too efficient reactive desorption mechanism (which releases $\mathrm{CH}_{3} \mathrm{OH}$ molecules in the gas phase upon formation on dust grain surfaces), atomic hydrogen tunneling or lack of physical evolution of the core in the model.

This work is part of the NOEMA large program SOLIS (Seeds of Life in Space), aimed at studying the formation of

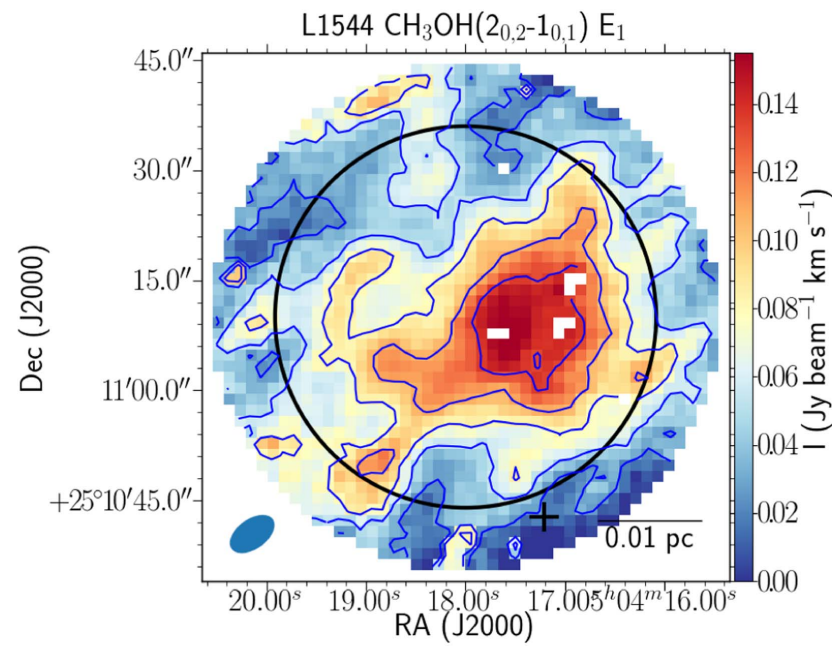

complex organic molecules at all stages of star formation (Ceccarelli et al. 2017).

The authors thank the anonymous referee for valuable comments that helped to improve the manuscript. The authors acknowledge the financial support of the European Research Council (ERC; project PALs 320620); A.P. acknowledges that partial salary support was provided by a CITA National Fellowship. I.J.-S. and D.Q. acknowledge the financial support received from the STFC through an Ernest Rutherford Fellowship and Grant (proposals number ST/L004801 and ST/M004139). Cecilia Ceccarelli acknowledges the financial support of the ERC (project DOC 741002).

\section{Appendix Additional Figures}

Here we present the maps of integrated intensities, velocity dispersions, centroid velocities, and local velocity gradients for the two weaker lines, $E_{1}$ and $E_{2}$.

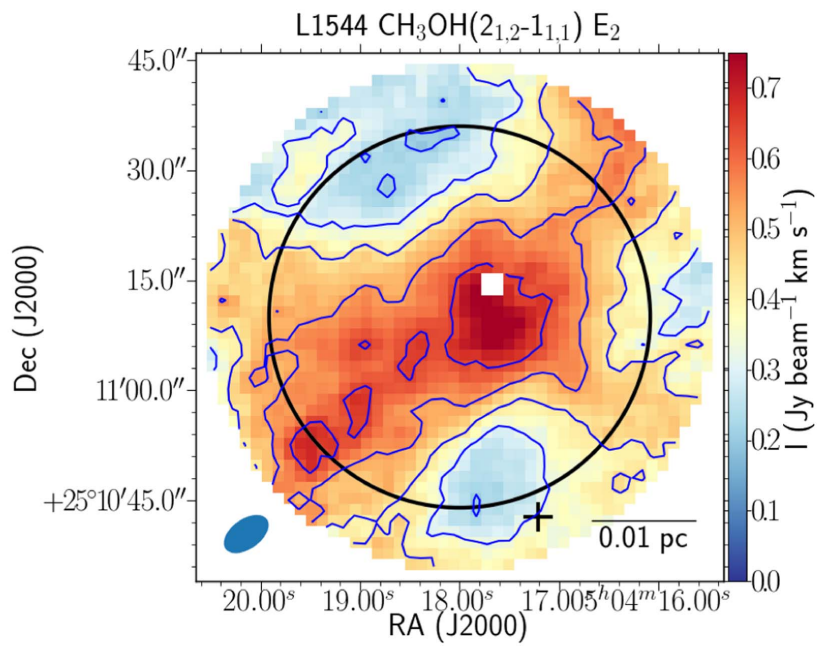

Figure 15. Integrated intensities of the methanol lines (NOEMA $+30 \mathrm{~m}$ ) for the $E_{1}(\mathrm{left})$ and $E_{2}$ (right) lines. The blue contours represent integrated intensity, and start at $0.027 \mathrm{Jy}$ beam ${ }^{-1} \mathrm{~km} \mathrm{~s}^{-1}$ with a step of $0.027 \mathrm{Jy}_{\text {beam }}^{-1} \mathrm{~km} \mathrm{~s}^{-1}$ for the $E_{1}$ line (left), and at $0.108 \mathrm{Jy} \mathrm{beam}{ }^{-1} \mathrm{~km} \mathrm{~s}^{-1}$ with a step of $0.108 \mathrm{Jy} \mathrm{beam}{ }^{-1} \mathrm{~km} \mathrm{~s}^{-1}$ for the $E_{2}$ line (right). $3 \sigma_{I}=0.005 \mathrm{Jy}_{\text {beam }}{ }^{-1} \mathrm{~km} \mathrm{~s}^{-1}$. The circle shows the primary beam of NOEMA. The cross shows the dust emission peak (Ward-Thompson et al. 1999). The synthesized beam of NOEMA is shown in the bottom left corner. The white pixels are those masked because of low-quality spectra.
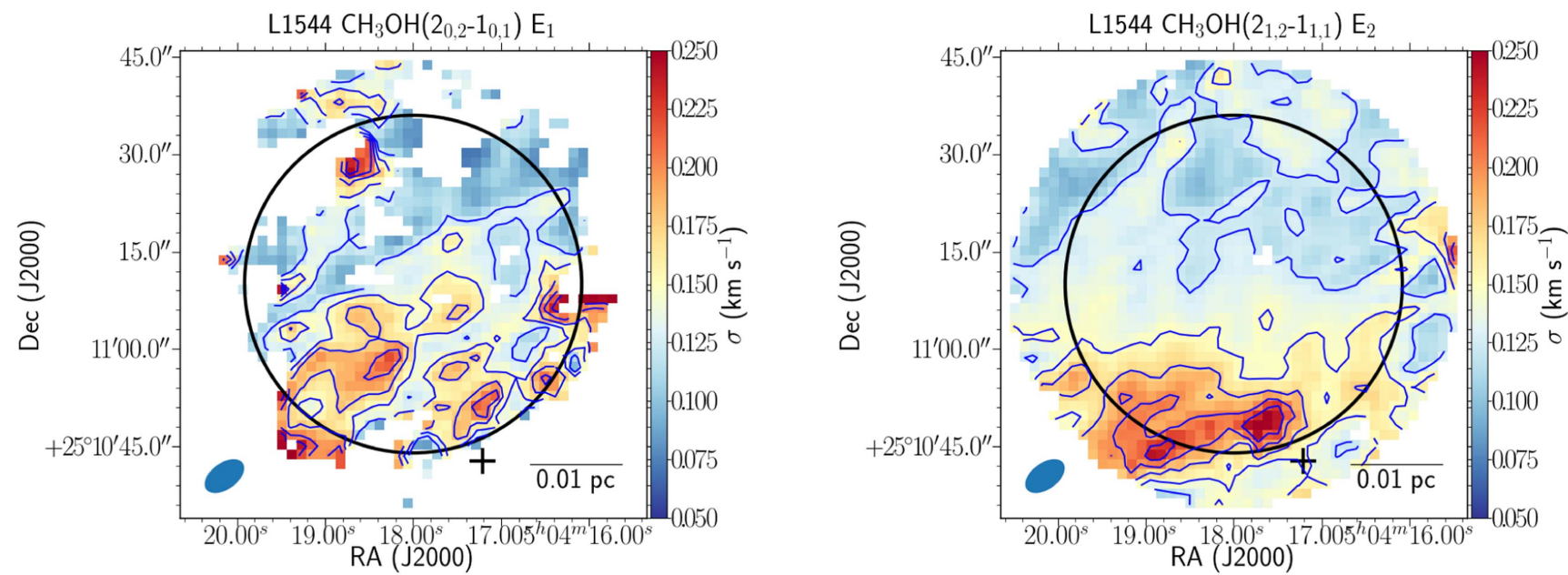

Figure 16. Velocity dispersions of the methanol lines for the $E_{1}$ (left) and $E_{2}$ (right) lines. The blue contours show velocity dispersions of $0.125,0.150,0.175,0.200$, and $0.225 \mathrm{~km} \mathrm{~s}^{-1}$. The circle shows the primary beam. The cross shows the $1.3 \mathrm{~mm}$ dust emission peak (Ward-Thompson et al. 1999). The synthesized beam is shown in the bottom left corner. The white pixels are those masked because of low-quality spectra. 

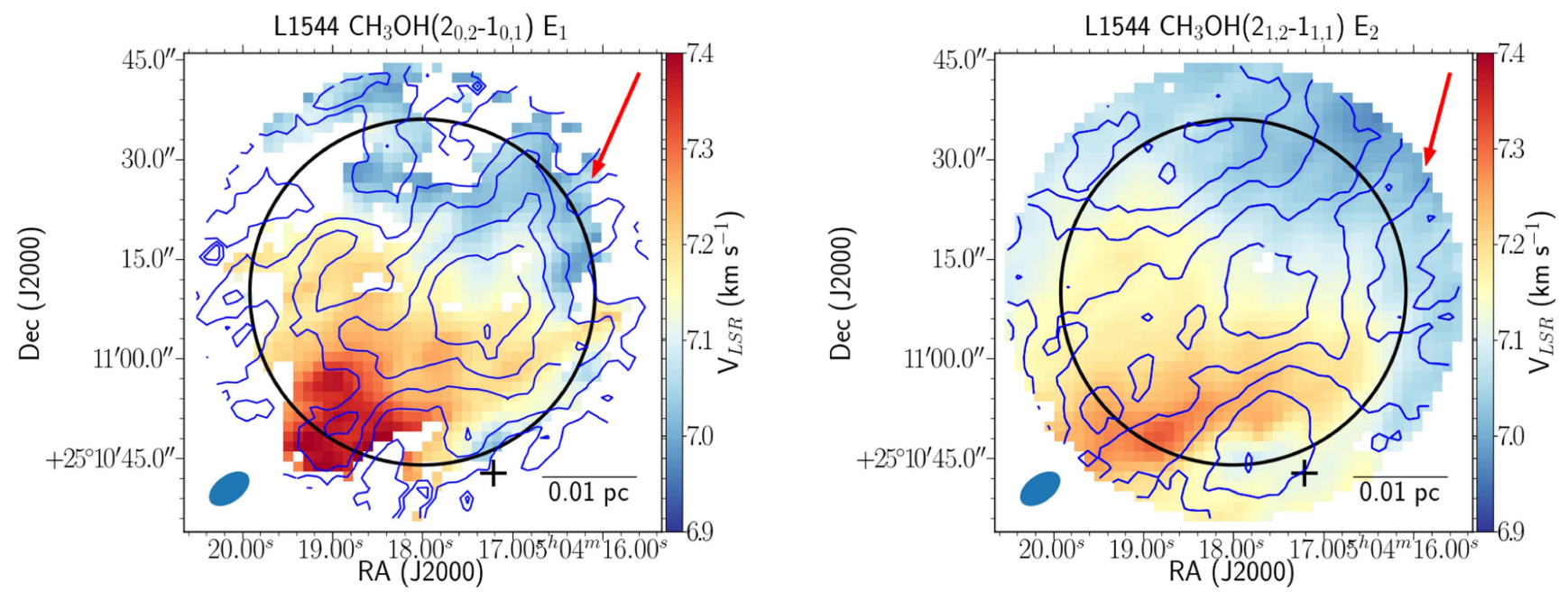

Figure 17. Centroid velocities of the methanol lines for the $E_{1}$ (left) and $E_{2}$ (right) lines. The blue contours represent integrated intensity, and start at $0.027 \mathrm{Jy}_{\text {beam }}{ }^{-1} \mathrm{~km} \mathrm{~s}^{-1}$ with a step of $0.027 \mathrm{Jy}_{\text {beam }}^{-1} \mathrm{~km} \mathrm{~s}^{-1}$ for the $E_{1}$ line (left), and at $0.108 \mathrm{Jy} \mathrm{beam} \mathrm{km} \mathrm{s}^{-1}$ with a step of $0.108 \mathrm{Jy} \mathrm{beam}^{-1} \mathrm{~km} \mathrm{~s}^{-1}$ for the $E_{2}$ line (right). $3 \sigma_{I}=0.005 \mathrm{Jy}_{\text {beam }}^{-1} \mathrm{~km} \mathrm{~s}^{-1}$. The red arrows show the total velocity gradients $\left(8.77 \pm 0.04\right.$ and $7.24 \pm 0.01 \mathrm{~km} \mathrm{~s}^{-1} \mathrm{pc}^{-1}$ for the $E_{1}$ and $E_{2}$ lines). The circle shows the primary beam. The cross shows the $1.3 \mathrm{~mm}$ dust emission peak (Ward-Thompson et al. 1999). The synthesized beam is shown in the bottom left corner. The white pixels are those masked because of low-quality spectra.
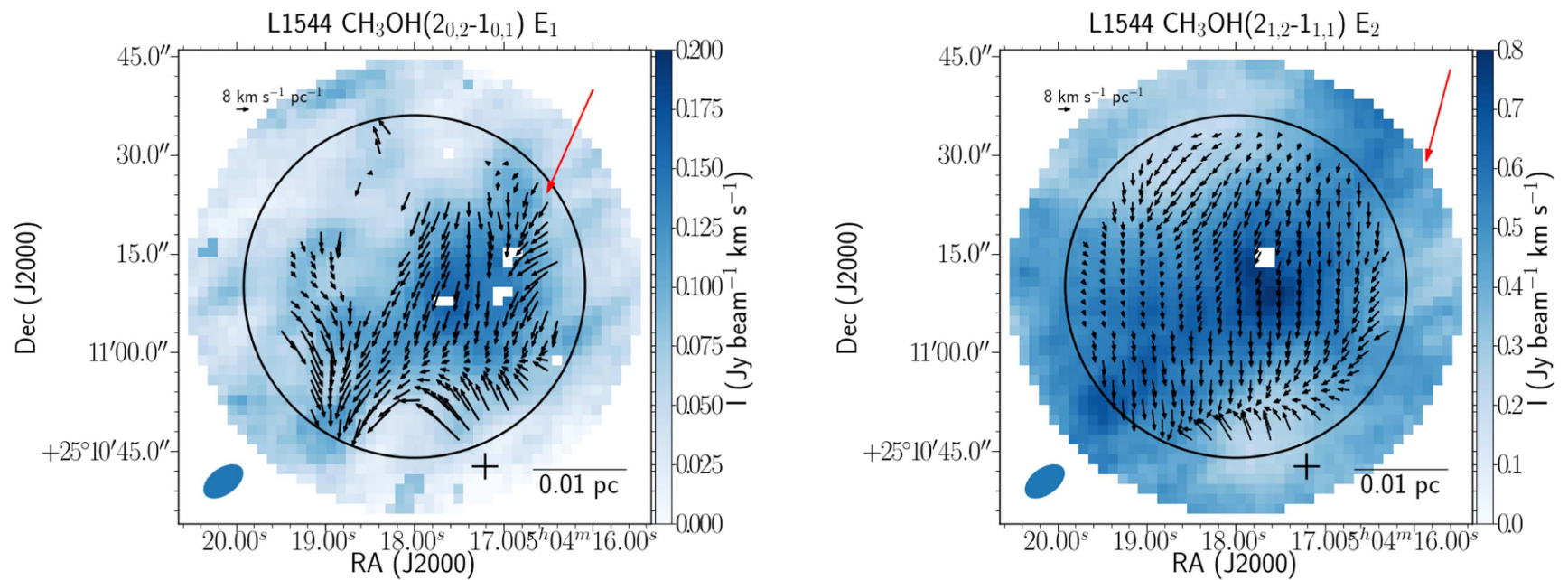

Figure 18. Local velocity gradients of the methanol lines for the $E_{1}$ (left) and $E_{2}$ (right) lines. The colorscale shows integrated intensity. The black arrows indicate the local velocity gradients. The red arrows show the total velocity gradients (the scale of the total gradients are eight times larger than the scale of the local gradients). The circle shows the primary beam. The cross denotes the $1.3 \mathrm{~mm}$ dust emission peak (Ward-Thompson et al. 1999). The synthesized beam is plotted in the bottom left corner. The white pixels are those masked because of low-quality spectra.

\section{ORCID iDs}

Anna Punanova (1) https://orcid.org/0000-0001-6004-875X Paola Caselli (i) https://orcid.org/0000-0003-1481-7911 Siyi Feng 1 https://orcid.org/0000-0002-4707-8409 Cecilia Ceccarelli (10 https://orcid.org/0000-0001-9664-6292 Roberto Neri (i) https://orcid.org/0000-0002-7176-4046 Francesco Fontani (10) https://orcid.org/0000-0003-0348-3418 Izaskun Jiménez-Serra (1) https://orcid.org/0000-0003-4493-8714 Luca Bizzocchi (1) https://orcid.org/0000-0002-9953-8593 Andy Pon (i) https://orcid.org/0000-0003-4612-1812 Anton I. Vasyunin (1) https://orcid.org/0000-0003-1684-3355 Pierre Hily-Blant i https://orcid.org/0000-0003-3488-8442 Leonardo Testi 1 https://orcid.org/0000-0003-1859-3070 Serena Viti (1) https://orcid.org/0000-0001-8504-8844 Felipe Alves (1) https://orcid.org/0000-0002-7945-064X Rafael Bachiller (1) https://orcid.org/0000-0002-5331-5386 Nadia Balucani (i) https://orcid.org/0000-0001-5121-5683
Claudio Codella (1) https://orcid.org/0000-0003-1514-3074 Cécile Favre (1) https://orcid.org/0000-0002-5789-6931 Jonathan Holdship (1) https://orcid.org/0000-0003-4025-1552 Claudine Kahane (10) https://orcid.org/0000-0003-1691-4686 Jake Laas 자 https://orcid.org/0000-0001-6876-6940 Ana López-Sepulcre (1) https://orcid.org/0000-00026729-3640

Yoko Oya (1) https://orcid.org/0000-0002-0197-8751 Jaime E. Pineda (1) https://orcid.org/0000-0002-3972-1978 Albert Rimola (1) https://orcid.org/0000-0002-9637-4554 Nami Sakai (ib https://orcid.org/0000-0002-3297-4497 Vianney Taquet (i) https://orcid.org/0000-0003-0407-7489

\section{References}

André, P., Men'shchikov, A., Bontemps, S., et al. 2010, A\&A, 518, L102 Bertin, M., Romanzin, C., Doronin, M., et al. 2016, ApJL, 817, L12 Bizzocchi, L., Caselli, P., Spezzano, S., \& Leonardo, E. 2014, A\&A, 569, A27 
Blake, G. A., Sutton, E. C., Masson, C. R., \& Phillips, T. G. 1987, ApJ, 315,621

Caselli, P., Benson, P. J., Myers, P. C., \& Tafalla, M. 2002a, ApJ, 572, 238

Caselli, P., Bizzocchi, L., Keto, E., et al. 2017, A\&A, 603, L1

Caselli, P., Walmsley, C. M., Tafalla, M., Dore, L., \& Myers, P. C. 1999, ApJL, 523, L165

Caselli, P., Walmsley, C. M., Zucconi, A., et al. 2002b, ApJ, 565, 344

Ceccarelli, C., Caselli, P., Fontani, F., et al. 2017, ApJ, 850, 176

Chacón-Tanarro, A., Caselli, P., Bizzocchi, L., et al. 2017, A\&A, 606, 142

Clemens, D. P., Tassis, K., \& Goldsmith, P. F. 2016, ApJ, 833, 176

Crapsi, A., Caselli, P., Walmsley, M. C., \& Tafalla, M. 2007, A\&A, 470, 221

Cruz-Diaz, G. A., Martín-Doménech, R., Muñoz Caro, G. M., \& Chen, Y.-J. 2016, A\&A, 592, A68

Garrod, R. T., \& Herbst, E. 2006, A\&A, 457, 927

Garrod, R. T., Wakelam, V., \& Herbst, E. 2007, A\&A, 467, 1103

Gibb, E. L., Whittet, D. C. B., Schutte, W. A., et al. 2000, ApJ, 536, 347

Ginsburg, A., \& Mirocha, J. 2011, PySpecKit: Python Spectroscopic Toolkit, Astrophysics Source Code Library, ascl:1109.001

Goldsmith, P. F., \& Langer, W. D. 1999, ApJ, 517, 209

Goodman, A. A., Benson, P. J., Fuller, G. A., \& Myers, P. C. 1993, ApJ, 406, 528

Hasegawa, T. I., Herbst, E., \& Leung, C. M. 1992, ApJS, 82, 167

Herbst, E., \& van Dishoeck, E. F. 2009, ARA\&A, 47, 427

Hily-Blant, P., Walmsley, M., Pineau Des Forêts, G., \& Flower, D. 2010 , A\&A, 513, A41

Jiménez-Serra, I., Vasyunin, A. I., Caselli, P., et al. 2016, ApJL, 830, L6

Kauffmann, J., Bertoldi, F., Bourke, T. L., Evans, N. J., II, \& Lee, C. W. 2008, A\&A, 487, 993

Keto, E., \& Caselli, P. 2008, ApJ, 683, 238

Keto, E., \& Caselli, P. 2010, MNRAS, 402, 1625

Keto, E., Caselli, P., \& Rawlings, J. 2015, MNRAS, 446, 3731

Keto, E., Rawlings, J., \& Caselli, P. 2014, MNRAS, 440, 2616

Keto, E., \& Rybicki, G. 2010, ApJ, 716, 1315

Lees, R. M., \& Baker, J. G. 1968, JChPh, 48, 5299

Maret, S., Ceccarelli, C., Tielens, A. G. G. M., et al. 2005, A\&A, 442, 527
Minissale, M., Dulieu, F., Cazaux, S., \& Hocuk, S. 2016a, A\&A, 585, A24

Minissale, M., Moudens, A., Baouche, S., Chaabouni, H., \& Dulieu, F. 2016b, MNRAS, 458, 2953

Müller, H. S. P., Thorwirth, S., Roth, D. A., \& Winnewisser, G. 2001, A\&A, 370, L49

Myers, P. C., Ladd, E. F., \& Fuller, G. A. 1991, ApJL, 372, L95

Parise, B., Castets, A., Herbst, E., et al. 2004, A\&A, 416, 159

Pickett, H. M., Poynter, R. L., Cohen, E. A., et al. 1998, JQSRT, 60, 883

Pon, A., Johnstone, D., Kaufman, M. J., Caselli, P., \& Plume, R. 2014, MNRAS, 445, 1508

Rimola, A., Taquet, V., Ugliengo, P., Balucani, N., \& Ceccarelli, C. 2014, A\&A, 572, A70

Schöier, F. L., Jørgensen, J. K., van Dishoeck, E. F., \& Blake, G. A. 2002, A\&A, 390, 1001

Shannon, R. J., Caravan, R. L., Blitz, M. A., \& Heard, D. E. 2014, PCCP, 16,3466

Spezzano, S., Bizzocchi, L., Caselli, P., Harju, J., \& Brünken, S. 2016, A\&A, 592, L11

Spezzano, S., Caselli, P., Bizzocchi, L., Giuliano, B. M., \& Lattanzi, V. 2017, A\&A, 606, 82

Tafalla, M., Mardones, D., Myers, P. C., et al. 1998, ApJ, 504, 900

Tafalla, M., Myers, P. C., Caselli, P., Walmsley, C. M., \& Comito, C. 2002 ApJ, 569, 815

Tafalla, M., Santiago-García, J., Myers, P. C., et al. 2006, A\&A, 455, 577

Tielens, A. G. G. M., \& Hagen, W. 1982, A\&A, 114, 245

Vastel, C., Caselli, P., Ceccarelli, C., et al. 2006, ApJ, 645, 1198

Vastel, C., Ceccarelli, C., Lefloch, B., \& Bachiller, R. 2014, ApJL, 795, L2

Vasyunin, A. I., Caselli, P., Dulieu, F., \& Jiménez-Serra, I. 2017, ApJ, 842, 33

Vasyunin, A. I., \& Herbst, E. 2013, ApJ, 769, 34

Vasyunin, A. I., Semenov, D., Henning, T., et al. 2008, ApJ, 672, 629

Vasyunin, A. I., Sobolev, A. M., Wiebe, D. S., \& Semenov, D. A. 2004, AstL, 30,566

Ward-Thompson, D., Motte, F., \& Andre, P. 1999, MNRAS, 305, 143

Watanabe, N., \& Kouchi, A. 2002, ApJL, 571, L173

Williams, J. P., Lee, C. W., \& Myers, P. C. 2006, ApJ, 636, 952

Xu, L.-H., \& Lovas, F. J. 1997, JPCRD, 26, 17 